\title{
Brillouin Distributed Fiber Sensors: An Overview and Applications
}

\author{
C. A. Galindez-Jamioy and J. M. López-Higuera \\ Photonic Engineering Group of the University of Cantabria, R\&D\&i Telecommunication Building, \\ Avenida Los Castros, 39005 Santander, Spain
}

Correspondence should be addressed to J. M. López-Higuera, higuera@teisa.unican.es

Received 16 April 2012; Revised 30 July 2012; Accepted 1 August 2012

Academic Editor: Romeo Bernini

Copyright ( 92012 C. A. Galindez-Jamioy and J. M. López-Higuera. This is an open access article distributed under the Creative Commons Attribution License, which permits unrestricted use, distribution, and reproduction in any medium, provided the original work is properly cited.

A review focused on real world applications of Brillouin distributed fiber sensors is presented in this paper. After a brief overview of the theoretical principles, some works to face the two main technical challenges (large dynamic range and higher spatial resolution) are commented. Then an overview of some real and on-field applications is done.

\section{Introduction}

Optical fibers have commonly used as communication channels where light waves propagate along haul distances. In this situation the fibers are isolated from the external perturbations by means of cabling techniques.

However, by enhancing the environment influences on the properties of the light that travels into the waveguide, the fibers can be used to detect, to monitor, and even to measure external perturbations (measurands) in an integral or distributed format [1].

When the optical power overpass a given power threshold, nonlinear phenomena such as Brillouin scattering can be forced inside the core fiber. Furthermore, Brillouin scattering of light in optical fibers can be used as a basis to develop optical devices such as fiber lasers [2], optical filters [3], and due to its strong dependence on environmental variables (strain and temperature), it is successfully employed in distributed fiber sensor systems [4].

In these cases, the optical fiber constitutes the medium where the interaction takes place, acting at the same time as distributed transducer and optical channel. These sensors are capable of measuring the change of a specific parameter (measurand) along the entire fiber transducer. Hence, the dynamic range (correlated with the maximum fiber-length of the transducer) and the spatial resolution (minimum fiberlength required to measure to consecutive perturbations or events) are key factors, whose values still remain as challenges (they must be improved).

\section{Background: Brillouin Frequency Shift in Monomode Optical Fibers}

The interaction between an electromagnetic wave and matter can generate variations in the molecular structure of the material. Classically, the incident light wave generates acoustic waves through the electrostriction effect (electrostriction is the tendency of materials to become compressed in the presence of an electric field) and induces a periodic modulation of the refractive index of material that provokes a light-backscattering like a Bragg grating. This scattered light is down-shifted in frequency due to the Doppler shift associated with the grating moving at the acoustic velocity. From the point of view of quantum physics, when the intensity of light can modify locally the density of the solid, a scattering process can appear. In this process the material absorbs part of the energy from the electromagnetic wave. This energy is used for generating a periodic structure, while the remaining energy is reemitted as a wave of lower frequency, provided that conditions of resonance between the light wave and the phonon are met. The scattering associated to this process was named as "Brillouin scattering" after Leon Brillouin did for the first time the theoretical description in 1910. 
Spontaneous scattering of light is mostly caused by thermal excitation of the medium, and it is proportional to the incident light intensity. On the other hand, the scattering process becomes stimulated if fluctuations in the medium are stimulated by the presence of another electromagnetic wave that reinforces the spontaneous scattering. The scattering process is in the stimulated regime provided that the intensity of the input light has a value above a level known as the threshold, which is lower than the threshold of the spontaneous regime. The stimulated scattering process is readily observed when the light intensity reaches a range between $10^{6}$ and $10^{9} \mathrm{Wcm}^{-2}$ [5] and is capable of modifying the optical properties of the material medium.

Stimulated Brillouin Scattering (SBS) can be achieved by using two optical light waves. In addition to the optical pulse, usually called the pump, a continuous wave (CW), the socalled probe signal, is used to probe the Brillouin frequency profile of the fiber [6]. A stimulation of the Brillouin scattering process occurs when the frequency difference of the pulse and the CW signal corresponds to the Brillouin shift and provided that both optical signals are counterpropagating in the fiber. The interaction leads to a larger scattering efficiency, resulting in an energy transfer from the pulse to the probe signal and an amplification of the probe signal.

The stimulated Brillouin interaction in single mode fibers can be modeled by the three-wave transient equations for the pump (subscript $p$ ) and Stokes (subscript $s$ ) waves with the field amplitudes $E_{p, s}(z, t)$ interacting with the acoustic wave $E_{a}(z, t)$ (in time $t$ and position $z$ along the fiber) [7]:

$$
\begin{gathered}
\frac{\partial E_{p}}{\partial z}-\frac{n_{f}}{c} \frac{\partial E_{p}}{\partial t}=E_{a} E_{s} \\
\frac{\partial E_{s}}{\partial z}+\frac{n_{f}}{c} \frac{\partial E_{s}}{\partial t}=E_{a}^{*} E_{p} \\
\frac{\partial E_{a}}{\partial z}+\Gamma E_{a}=\frac{1}{2} \Gamma_{1} g_{B} E_{p} E_{a}^{*} \\
\Gamma=\Gamma_{1}+i \Gamma_{2},
\end{gathered}
$$

where $n_{f}$ is the fiber refractive index, $c$ is the velocity of light in vacuum, and $g_{B}$ is the Brillouin gain factor. $\Gamma$ is the damping constant of the acoustic wave, $\Gamma_{1}$ is the damping time of the phonon, $\Gamma_{2}$ is the detuning angular frequency given by $\Gamma_{2}=2 \pi\left(\nu-\nu_{B}\right)$ and $\nu$ is the beat frequency between the probe wave and the pump wave. The sensor system focuss on measuring the Brillouin backscattered light, which gives information about changes in temperature or strain experienced by the fiber. Considering pump pulses larger (time-width) than the phonon lifetime and no pump depletion, the Brillouin backscattered light power $P_{B}(z, v)$ detected at the receiver can be given by $[5,7]$

$$
\begin{gathered}
P_{s}(z=0, v, t)=\left|E_{s}\right|^{2}, \\
P_{S}=g_{B}\left(\nu, \nu_{B}\right)\left(\frac{c}{2 n}\right) P(t) \exp (-2 \alpha z), \\
g_{B}=g_{0} \frac{\left(\Delta \nu_{B} / 2\right)^{2}}{\left(\nu-\nu_{B}\right)^{2}+\left(\Delta \nu_{B} / 2\right)^{2}}, \\
g_{0}=\frac{2 \pi n^{7} p_{12}^{2}}{\lambda_{p}^{2} \rho_{0} v c \Delta \nu_{B}}
\end{gathered}
$$

where $P(t)$ is the total power of the launched pulsed light, $\alpha$ is the attenuation coefficient of the fiber, $p_{12}$ is the electooptic coefficient, $v$ is the acoustic velocity, $\lambda_{p}$ is the pump wavelength, $\rho_{0}$ is the material density, and the Brillouin gain spectrum $g_{B}$ in this case is given by a Lorentzian function and is assumed not to depend on $z$. The parameter $\nu_{B}$ is the frequency at which $g_{B}$ has a peak value $g_{0}$, and $\Delta v$ is the full width at half-maximum (FWHM). Also, it is important to remark that there is an exponential relationship between the Brillouin power gain and the Brillouin gain spectrum, which is maximized at $\nu_{B}$ value.

According to the mathematical description presented in (1), Brillouin scattering is strongly dependent on thermodynamical variables [8-12]. The dielectric constant varies according to the pressure wave that is generated and which travels along the medium. Then, the Brillouin shift frequency is a function of the acoustic phonon, as well as the medium structure and its constituents. The material structure is clearly perturbed by changes on environmental temperature or by strong alterations on its density distribution; such is the case when a longitudinal force or a stress is applied. Those transversal or longitudinal forces relay on a shrinking or an enlargement of the original size of the material.

The Brillouin frequency shift has a linear dependence (for values of strain and temperature within its tolerance ranges) on the applied strain $\varepsilon$ and the temperature variation $\Delta T$ (at a reference temperature $T_{0}$ ) that can be written as $[8,9]$

$$
\nu_{B}(T, \varepsilon)=C_{\varepsilon} \Delta \varepsilon+C_{T} \Delta T+\nu_{B}\left(T_{0}, \varepsilon_{0}\right)
$$

where $C_{\varepsilon}$ is the strain coefficient $(\mathrm{MHz} / \mu \varepsilon), C_{T}$ is the temperature coefficient $\left(\mathrm{MHz} /{ }^{\circ} \mathrm{C}\right)$ and reference strain $\varepsilon_{0}$. These values are mostly determined by the fiber composition, pump wavelength, fiber coatings, and jackets.

Brillouin scattering was proposed for the first time to measure temperature in 1989 [13], and, currently, it is widely used for distributed temperature and strain sensing because the Stokes side-lobe is temperature and strain dependent. One reason of this success is that Brillouin effect can be used in long transducers (hundreds of kilometers) made of standard monomode telecommunication optical fibers, since the Brillouin frequency shift is about $10-11 \mathrm{GHz}$ at $1550 \mathrm{~nm}$ [4]. An example of this dependence for a standard single mode fiber (SMF) is shown in Figure 1. 


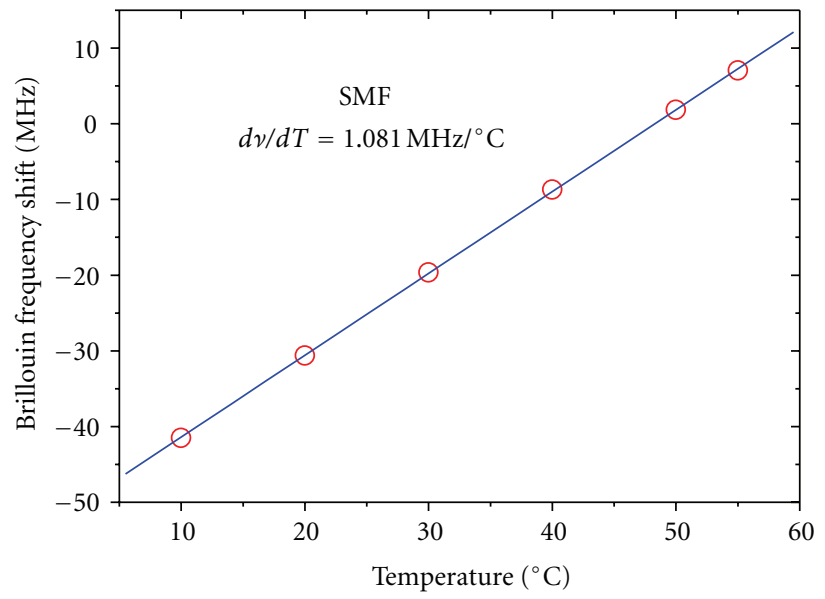

(a)

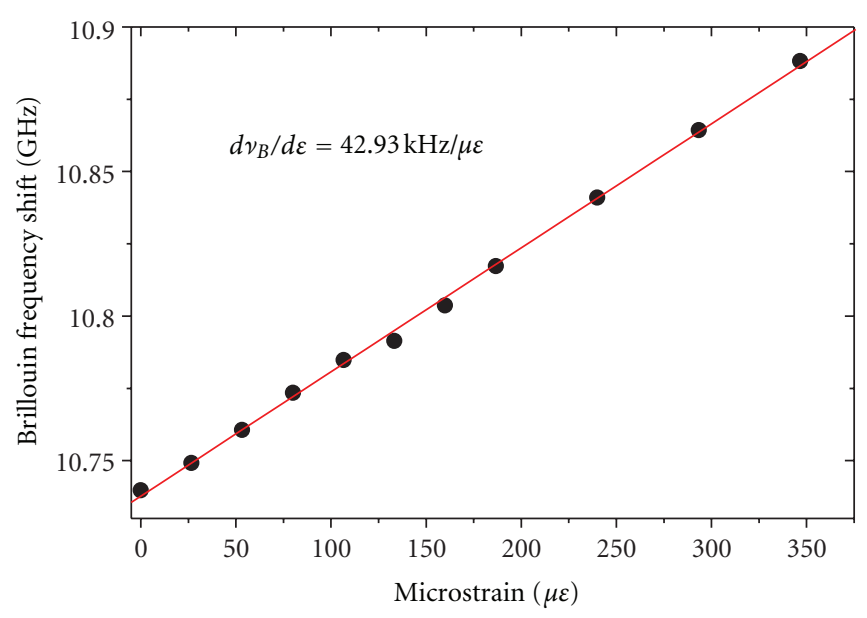

(b)

Figure 1: Experimental data of Brillouin shift dependence on the temperature and strain.

\section{Brillouin Scattering Distributed Fiber Sensors: Quick Overview}

A device or a system capable of detecting, measuring, and reproducing faithfully a particular physical or chemical variable (measurand) in the electrical domain may be recognized as a sensor or a sensor system [1]. If light is used in such sensors, and the measurand changes some of the light properties, the device is known as photonic or optical sensor. These changes on the light properties usually happen in the transducer part of the sensor. Within the photonic sensors, the fiber sensors are those who are made up with optical fibers and the technology around them [1]. Distributed fiber optic sensors are capable of detecting and measuring variables along a fiber that acts both as a distributed transducer and as an optical channel. Some examples of sensor fibers are shown in Figure 2, in this case three fibers, one for strain sensing, one for temperature sensing, and another for simultaneous strain and temperature sensing, are pictured (picture courtesy of the Photonic Engineering Group of the University of Cantabria).

As it was previously mentioned, Brillouin scattering is a phenomenon that strongly depends on the temperature in the medium, its entropy, and the material density. In most of the cases, these variables affect the frequency shift or the Brillouin spectrum linewidth. Thus, this dependence can be used for indirectly determining the influence of external or intrinsic variations in the medium where the scattering takes place. Additionally, the Brillouin shift process can be accurately $(\sim \mathrm{cm})$ localized along the fiber, by time domain or frequency correlation techniques, among others. Hence, Brillouin scattering of light in optical fibers can be used as a basis to develop accurate distributed optical sensors and optical devices. In these cases, the optical fiber constitutes the medium where the interaction takes place, acting at the same time as distributed transducer and optical channel. These sensors are able to measure the change of a specific parameter along the entire fiber transducer within a given certitude.

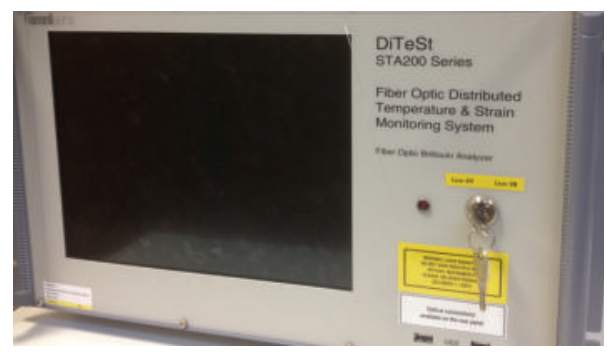

(a)

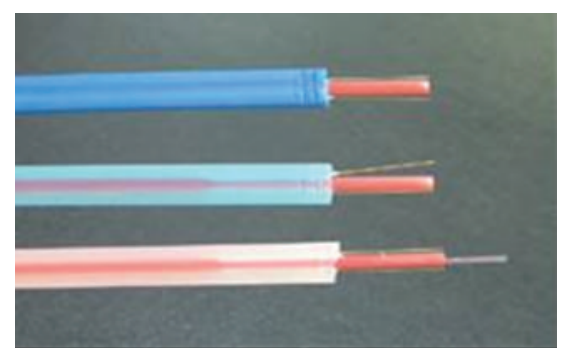

(b)

FIGURE 2: Illustration of one optoelectronic (a) and three samples of fiber transducers for both temperature and strain (b) of a stimulated Brillouin distributed sensing system, courtesy of the Photonic Engineering Group of the University of Cantabria.

Prior to talk about the sensor schemes and ultimate ranges, it is important to clarify the meaning of some key parameters commonly used to characterize the distributed fiber sensors, such as spatial resolution, dynamic range, and distance range.

The minimum change in the measurement, meaningfully detectable by the measurement system, is the resolution. In an optical fiber the minimum distance between two step transitions of the measurand is the spatial resolution. The maximum cumulated one-way (or two-way) loss in the optical link between the interrogator and the measurement point that makes possible a measurement within a specified 
performance is the dynamic range, which is measured as the ratio of the difference between the extremes of the measurement range to the resolution (in $\mathrm{dB}$ ). The fiber length over which the measurement can be performed within the stated uncertainty and spatial resolution is denoted as distance range.

From a general point of view Brillouin sensors can be classified in two main kinds: spontaneous Brillouin sensors and stimulated Brillouin sensors. The methods where only the incident light is launched into the optical fiber and there is not any additional stimulus on the phonon generation are denoted as spontaneous Brillouin sensing configurations [14-18]. Coherent detection of spontaneous Brillouin scattering gives greater dynamic range and allows simultaneous measurements of temperature and strain. However, nonlinear effects that limit the input probe pulse power, the weakness of the Brillouin signal, and the two-way fiber loss $(0.4 \mathrm{~dB} / \mathrm{km})$ lead to a rapid reduction in performance as the sensing ranges are extended beyond $50 \mathrm{~km}$. Brillouin optical time domain reflectometry (BOTDR) $[19,20]$, the Landau-Placzek ratio [21] method, and Brillouin optical correlation domain reflectometry (BOCDR) [22] are part of this classification. On the other hand, Stimulated Brillouin sensors are based in the additional stimulus on the phonon generation given by an additional incident light, that is, the Brillouin scattering is enhanced or amplified.

BOTDR is a coherent detection method that uses a pulsed light. This light is launched into the optical fiber to generate spontaneous Brillouin scattering. As illustrated in Figure 3, the backward light is measured with a coherent receiver by mixing the scattering signal with that from a local oscillator $[19,20]$. Since the power in the backscattered signal is small, fiber attenuation can induce a negative effect on the quality of the measurement. To compensate this drawback a coherent detection is currently used. In Brillouin optical time domain reflectometry the backscattered light, which is generated by the pump light launched in a fiber, is combined with a local oscillator. Nonetheless its dynamic range decreases with the length of the fiber. More disadvantages on this method are the fact that the spatial resolution cannot be less than one meter, the frequency shift is simultaneously dependent on both the temperature and the longitudinal strain, and that is necessary to introduce electrical filtering to eliminate Rayleigh signal.

Since the Brillouin frequency shift is a function of temperature and strain simultaneously, there is no way, uniquely measure each variable unless their effects can be separated. Nonetheless, the ratio between the intensities of the Rayleigh and Brillouin backscattered light (LandauPlaczek ratio (LPR)) is only dependent on temperature, and it can be used for obtaining a distributed temperature profile from a fiber regardless of the strain distribution [21]. The standard OTDR technique is used for determining the fiber loss profile as a function of position, later this is subtracted from the intensity measurements made with the BOTDR system and the intensity measurements are cleaned out of fiber attenuation and any form of fiber losses.

The Brillouin shift on BOCDR is measured by controlling the interference of the continuous probe and pump

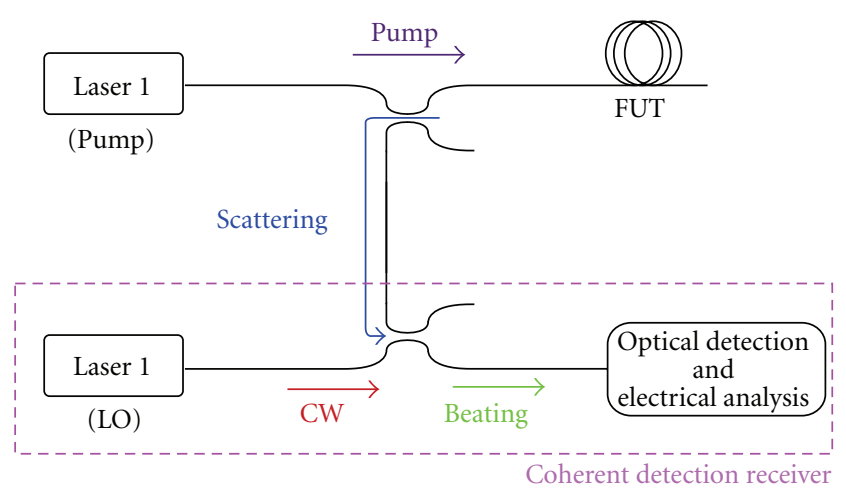

FIgure 3: Typical configuration for a BOTDR system. FUT is the fiber under test or distributed transducer, LO is the local oscillator, and $\mathrm{CW}$ is the continuous wave.

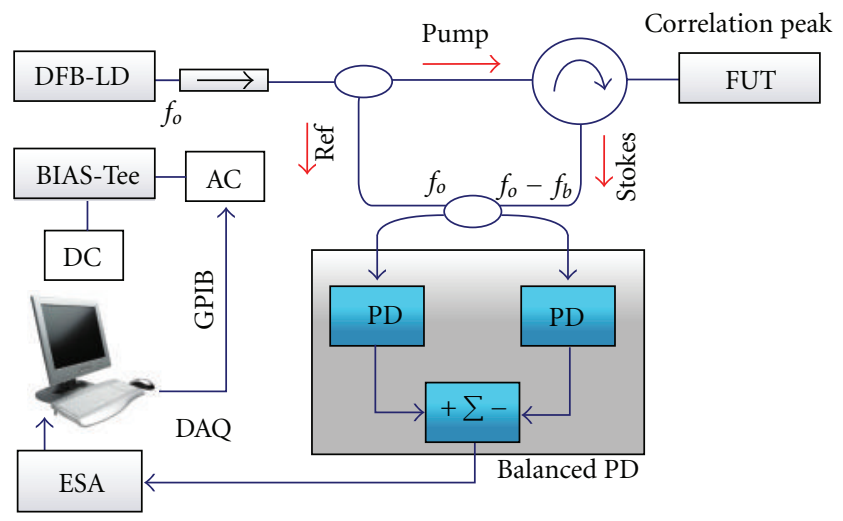

FIGURE 4: Schematic description of a Brillouin optical correlation domain reflectometry.

waves within the optical fiber, and the position is determined by scanning along the fiber. This technique allows the spatial range to be below the one meter range along one kilometer of optical fiber [23]. Since BOCDR is not based on an optical pulse but on a continuous wave, long integration time for the reflected signal is not needed, hence it is faster than BOTDR. However, the number of sample points on the fiber is limited by the spatial resolution and the spatial range. An experimental setup is depicted in Figure 4.

BOTDA uses Brillouin gain (or loss) spectroscopy in which a pulsed-optical wave (pump) and a counterpropagating light (probe/Stokes), normally a continuous wave, are injected into an optical fiber; the two most frequently implementaed ways are shown in Figure 5. When the frequency difference between the pulsed and the continuous light $\left(\Delta v=v-v_{B}\right)$ is tuned to the Brillouin frequency $\nu_{B}$ of the fiber, the continuous light is amplified through the stimulated Brillouin scattering process; that is, the increasing continuous light is measured as function of time, like with OTDR [24] (Figure 6). The gathered signal using the BOTDA technique is higher than the Rayleigh backscattering power, for example, when they launched continuous light power into standard fibers which is around 


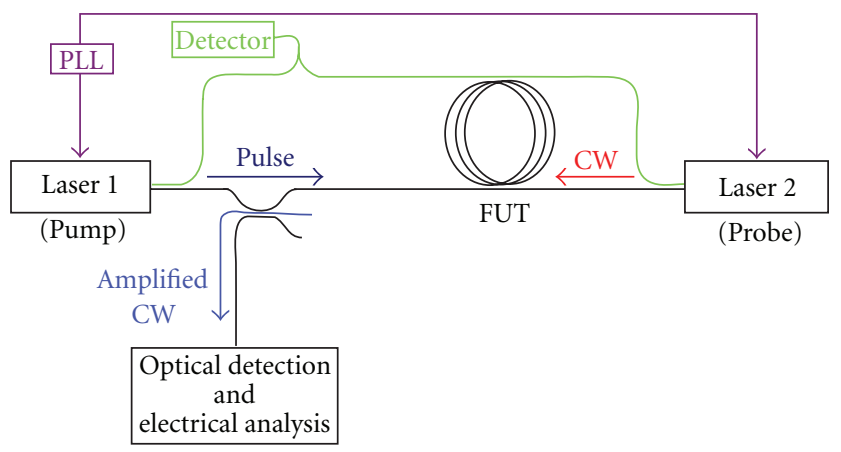

(a)

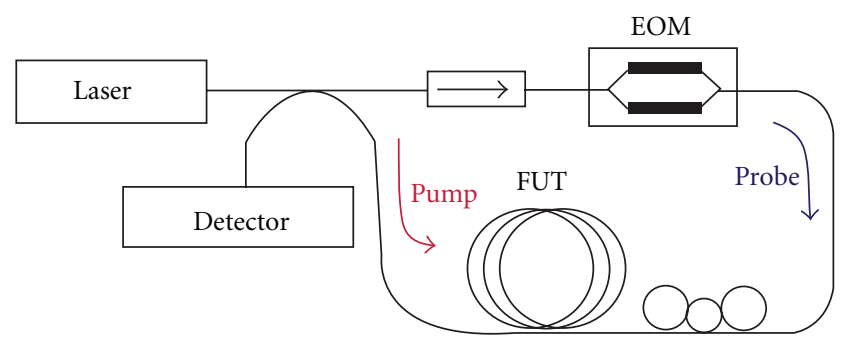

(b)

Figure 5: Experimental setup for BOTDA, using two sources (a) and one source (b).

$0.1 \mathrm{~mW}$, the backscattering can be a hundred times higher than the Rayleigh [8].

The pump and probe signals are launched into opposite ends of the fiber in order to enhance the scattering process. Thus the Brillouin gain spectrum is depicted by each value of the frequency difference, $\Delta \nu$, and is given by $(2 \mathrm{a})$ and (2b). Effects on the pump-probe technique have been widely studied, such as the Fresnel reflection [25], electrooptical modulation effects $[26,27]$, or pulse shape effects $[28,29]$.

In BOTDA based on pulse pumping, spatial resolution is limited to $\sim 1 \mathrm{~m}$ due to the finite phonon lifetime [5, 7]. To overcome this constraint, several techniques that include variations on the probe and handling the pumping (or probe) intensity have been proposed. In the timedomain pumping schemes, the more significant methods are (1) prepumping [30, 31], (2) differential pulse-width pairs (DPP-BOTDA) with or without phase shift [32-35], (3) dark pulses [36] (by these methods, $\mathrm{cm}$ or even mm scale spatial resolution has been demonstrated $[37,38]$ ), and (4) dynamic Brillouin gratings (DBGs) [39].

The mentioned methods achieve the goal of simultaneously high spatial and spectral resolution thanks to an acoustic field preactivated by a low intensity prepump pulse [3141] or a continuous pump background [42] (see Figure 7). Even, when the pump is restored to a nonnull intensity after the pulse, it interacts with the decaying acoustic wave, producing "echoes" in the acquired signal [35]. Although the acoustic wave partially decays during the pulse duration, and a second attenuated response appears beyond the acoustic lifetime, the measurement of submeter perturbations on the fiber may be hidden. One way to suppress or attenuate the impact of this background Brillouin response is based on the subtraction of two Brillouin gain spectra obtained from pulses shifting in time or pulses with different widths [34]; in this technique, the rising and the falling times of the pulses define the spatial resolution (see Figure 8). Another proposal to overcome the appearance of ghost peaks in the Brillouin gain spectra, which makes ambiguous the determination of the Brillouin frequency shift, is addressed to the numerical correction algorithms applied to BOTDA measurements, such as the correction method based on the deconvolution of the time traces, in which each trace is inverse filtered by use of the fiber response evaluated in the resonance case [35] or the iterative numerical approaching, using the analytical model of the stimulated Brillouin scattering in the frequency domain [43].

BOTDA systems require a uniform signal-to-noise ratio (SNR) to avoid uneven Brillouin gain along the optical fiber. Thus, a low Brillouin gain across a long sensing system is needed. One compromise of low gain system over a long sensing fiber causes an increment of the spatial resolution. In order to maintain the SNR high and a low Brillouin gain, the pump power should be kept minimized and the probe power maximized, but below the influence if the modulation instability [44]. The modulation instability is a process induced mostly by the amplitude and phase modulation part, which is included in BOTDA systems to generate the probe from the same coherent pump source. To avoid the effect of the modulation instability, a dispersion shifted fiber with normal dispersion can be used [45], by coding the pulse [46] 


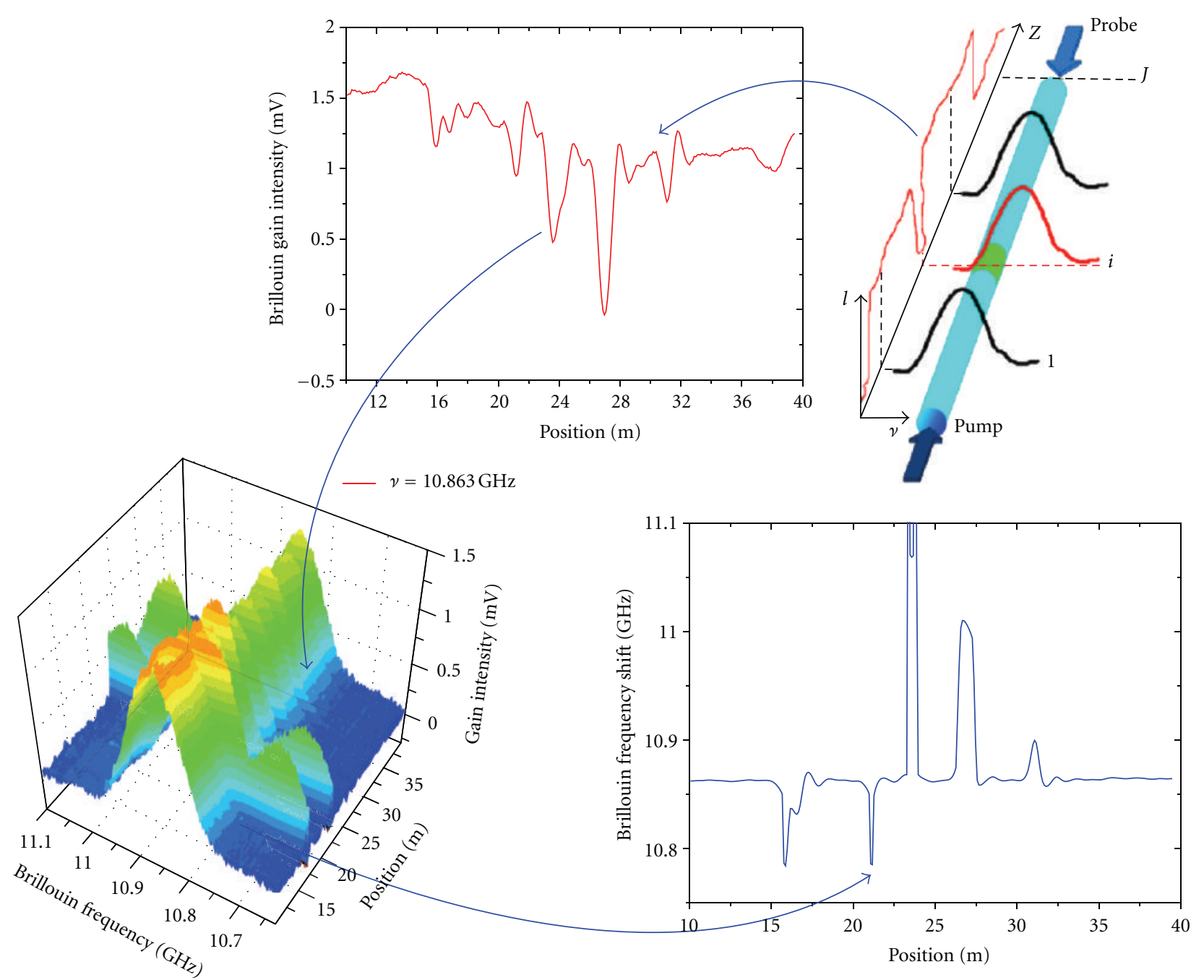

FIGURE 6: Traces and fiber distribution of the Brillouin frequency shift.

or an unbalanced double sideband probe [47]. The coding technique could reduce the spatial resolution, $25 \mathrm{~cm}$ or $2 \mathrm{~m}$ over a long distance range $60 \mathrm{~km}$ and $120 \mathrm{~km}$, respectively $[48,49]$. Additionally to these techniques, an interesting solution to enhance the spatial range without compromising the SNR ratio is addressed to the combination of techniques, or hybrid sensors, such as the Brillouin sensing with Raman amplification [50] to minimize the modulation instability.

The measurement time required by a traditional BOTDA system is on the order of minutes [51], time that also depends on the total length range and constitutes a serious drawback for dynamic detection in health monitoring structures. Dynamic variations of temperature or strain can be measured using Brillouin sensors by modifying the sensor technique or the system. The simplest method consists of the direct measurement of the intensity of the Brillouin peak gain/loss signal versus time, allowing the use of a BOTDA system $[52,53]$, which drastically reduces the measurement time, but it has a threshold detection and requires high averaging sampling as the length range is enlarged. Similar to this idea is the technique that uses two counter-propagating optical pulses with a fixed optical frequency difference; this difference is set to a spectral distance from the local Brillouin frequency shift approximately equal to half the Brillouin gain spectrum linewidth, then any vibration-induced modulation of the local Brillouin frequency shift will be measured as an intensity variation of the Stokes pulse peak intensity [54]; important changes in the setup are required in this technique and nonperiodic dynamic variations are not detected. Another method uses the polarization dependence of the Brillouin gain to avoid the need for scanning the pumpprobe frequency shift [55]. To reduce the measurement time, a technique based on a multitude of pump signals in the form of a frequency-domain comb in a complex BOTDA based system is also proposed [56]. Further to these techniques, a technique for measuring dynamic variations of temperature or strain based on the well-known anomaly detection method referred to as the RX algorithm to process the data gathered from a BOTDA system [57] was presented. This technique exploits the Brillouin sensing advantages without punishing complexity or performance limitations. Most recently a method to enhance the time for measuring 


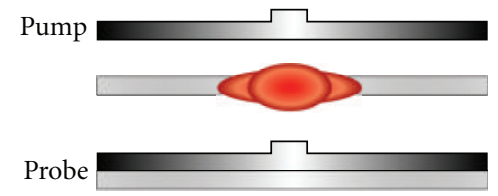

(a)

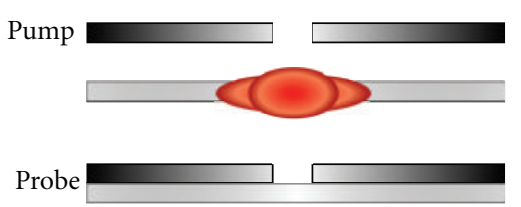

(b)

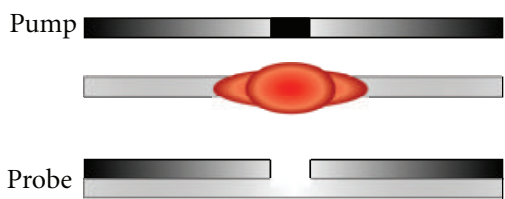

(c)

Figure 7: (a) Configuration of bright pulse, (b) configuration of dark pulse, and (c) configuration of phase pulse.

the Brillouin frequency detection by generating a comb probe was proposed [58]; this method uses an arbitrary waveform generator (AWG) in the intensity modulation stage.

Proposed by Hotate [39], the BOCDA is a technique that controls Brillouin scattering using the synthesis of optical coherence function (SOCF). This technique involves phase modulation of the continuous pump wave and the probe wave. Brillouin interaction only takes place in positions where the phase of these two signals is highly correlated (Figure 9). As the phase difference between the pump and probe is changed, the position along the fiber changes where the Brillouin interaction occurs; thus providing a way to scan the length of the fiber. In conventional BOTDA techniques, if the spectral width of the pulse exceeds the Brillouin linewidth, the gain spectrum broadens and the measurement deteriorates. Nonetheless, in BOCDA if the spectral width of the pump and probe increases, the resolution of correlation technique also increases. Spatial resolution up to $1.6 \mathrm{~mm}$, as well as improvements on the sampling rate of $1 \mathrm{kHz}$, and measurement range of $1 \mathrm{~km}[59,60]$ were achieved.

A common drawback of the BOCDA system is its limited measurement range due to the periodic nature of sensing position and that the transducer length is shorter than other Brillouin-based sensors; additionally the transducer is much more complex and the postprocessing is more intense, which may affect negatively the measurement time.

Brillouin optical frequency domain analysis (BOFDA) is based on the measurement of a complex transfer function that relates the amplitudes of counter-propagating pump and probe waves along a fiber [61]. The continuous probe wave is modulated in intensity with a sinusoidal signal over a range of frequencies, whilst in the pump wave an intensity modulation is induced. This induced signal has

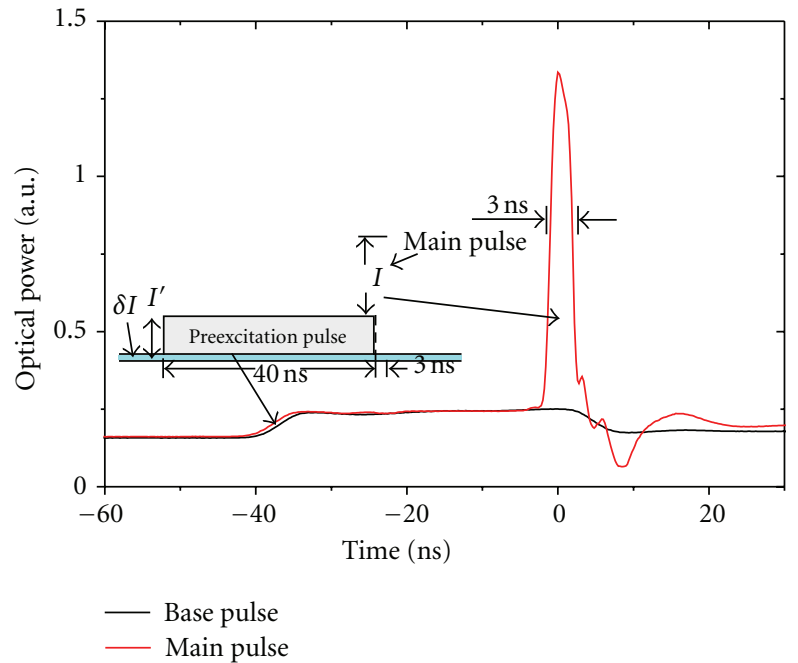

(a)

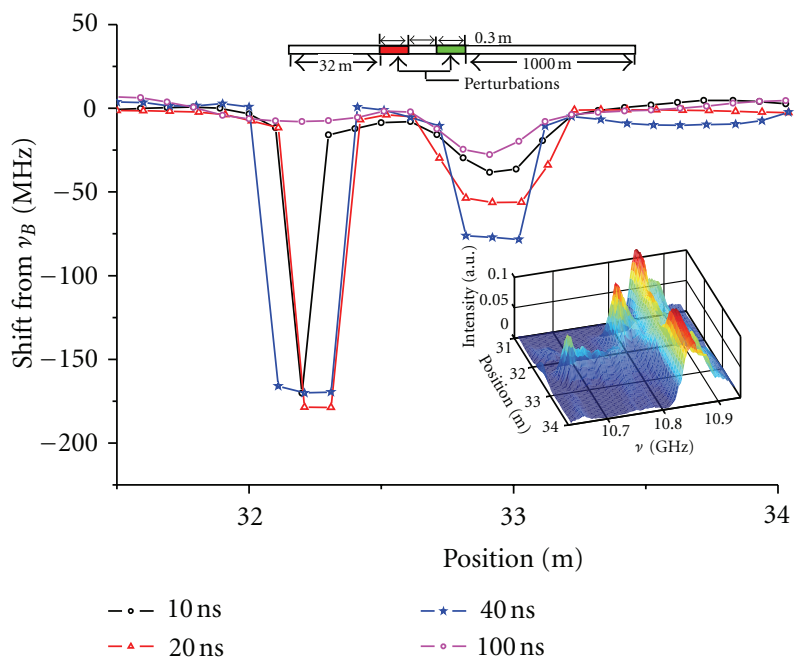

(b)

FIGURE 8: The pump light is basically formed by a pump-prepump pair pulse, and by subtracting the couple Brillouin backscattering it is possible to use larger base pulses to obtain stronger backscattered signals or to reach larger distances in the fiber. (a) Experimental data of preexcitation optical pulse. (b) Experimental data for $2 \mathrm{~ns}$ of main pulse and four values of P-pulse. Brillouin spectrum gain (inset figure).

an alternating component (AC component/part) due to the interaction with the counter-propagating probe wave. By measuring the changes in the AC pump wave component the Brillouin frequency shift profile is determined via the complex base-band transfer function. Once the base band transfer function is determined, the impulse response is calculated by applying the inverse Fourier transform (IFFT) to the function (Figure 10). Thus the temperature or strain can be determined from the Brillouin profile along the fiber $[62,63]$.

Recently, the concept of Brillouin dynamic grating (BDG) has been newly implemented in polarization maintaining fibers (PMFs) [64] and single mode fibers [65], since 


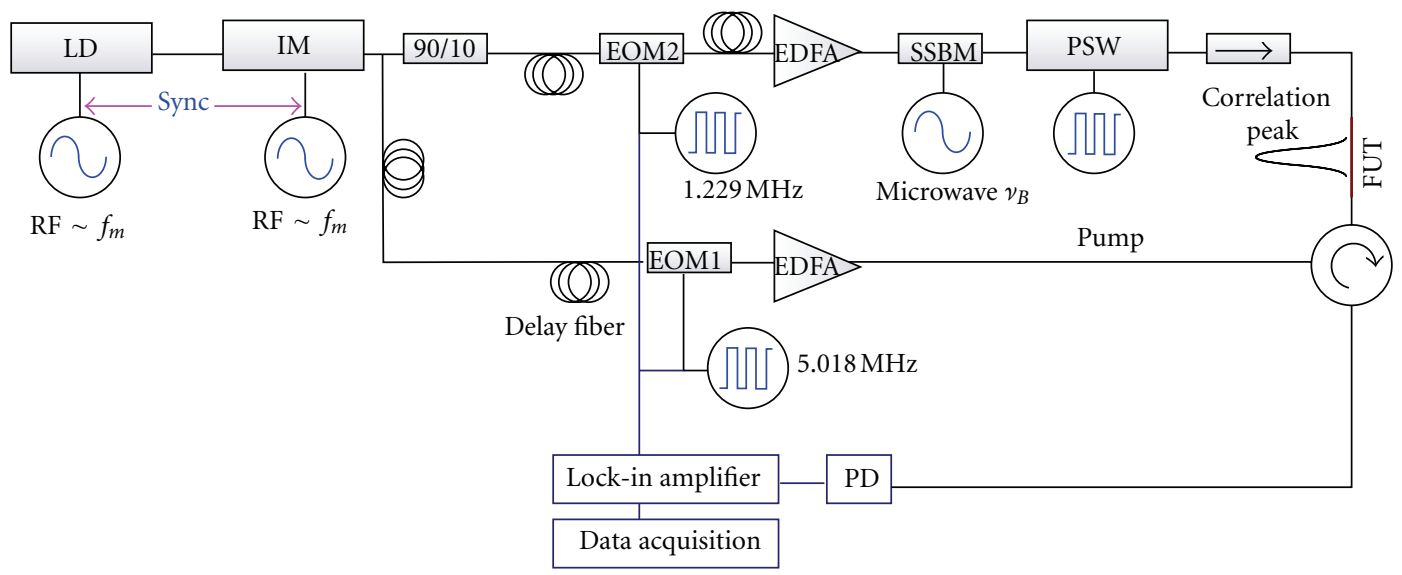

FIGURE 9: Experimental setup for BOCDA technique.

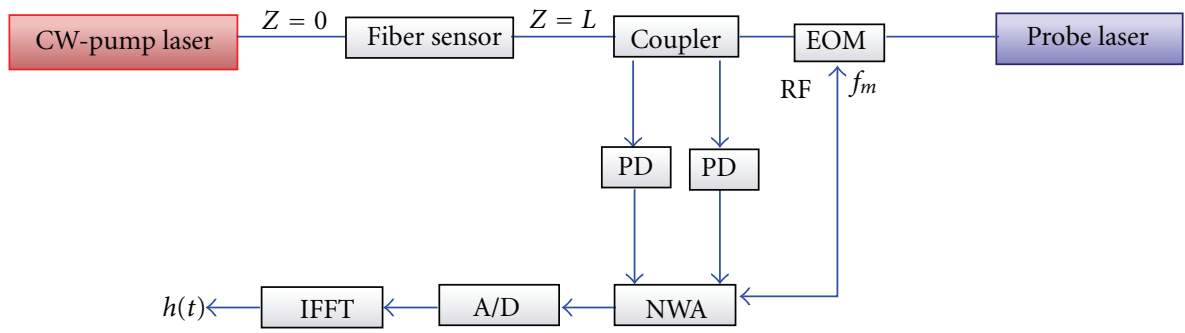

Figure 10: Basic schematic configuration of a BOFDA sensor system.

the operation principle strongly depends on the local birefringence of the medium. Acoustic waves generated during the process of stimulated Brillouin scattering in one polarization are used to reflect an orthogonally polarized wave (probe wave) at a different optical frequency from the pump. Figure 11 shows the conceptual scheme of the BDG; the frequency separation $\Delta f$ between the pump and the probe waves in the BDG operation is determined by the local birefringence of the fiber and lies in the several tens of $\mathrm{GHz}$ in usual cases. BDG has been used as strain and temperature sensors $[66,67]$, birefringence sensor [68] or as tunable delay lines [69]. BDG can also be applied to enhance the spatial resolution of an ordinary Brillouin optical timedomain analysis (BOTDA) system by replacing the Brillouin probe with the reflection from the BDG [70].

\section{Some Field Significant Applications}

Distributed fiber sensing is a really attractive technique for structural health monitoring (SHM) [71], since it can provide information of strain and temperature about a section or the complete structure with noise electromagnetic immunity, in hazardous environments, with durability, robustness, measurement reliability, stability, good strain and temperature sensitivity $\left( \pm 10 \mu \varepsilon, \pm 1^{\circ} \mathrm{C}\right)$, long range measurement $(\sim 100 \mathrm{~km})$ and suitable spatial resolution (couple centimeters minimum) to diverse structures and shapes. Despite it is a promising diagnostic tool, it is not easy to implement outside laboratories, because each application is a unique project by itself. Each application provides new challenges on the Brillouin sensing technique adaptation and standards, as well as the transducer implementation, ranges of measurement, calibration and environmental conditions.

Typically when review articles about the distributed Brillouin sensing technique are published, authors always refer only to the laboratory implementation, but none talks about the real world challenges of the technique, that is, why in this section a review of some interesting field applications is presented, to appreciate how this technique can be adapted no matter its operating principle.

4.1. In Civil Infrastructures. A few examples of the use of Brillouin distributed sensors in infrastructures such as bridges, railways, and land monitoring are briefly commented in this section.

4.1.1. Bridges and Monitoring. Near-to-surface fiber (NSF) embedding and smart-FRP (fiber reinforced polymer) sensor bonding techniques have been experimented on small reinforced concrete (RC) bridges subject to a diagnostic load test [72]. Two bridges with similar dimensions were instrumented and tested under similar environmental conditions. Acryl cyanide and epoxy putty were used, respectively as bonding and encapsulating media for NSF. Being both fragile materials, the crack could easily propagate across the NSF section inducing a strain distribution on the sensing fiber that is mostly concentrated in the small distance between the crack edges. This very short step-like condition in the strain 


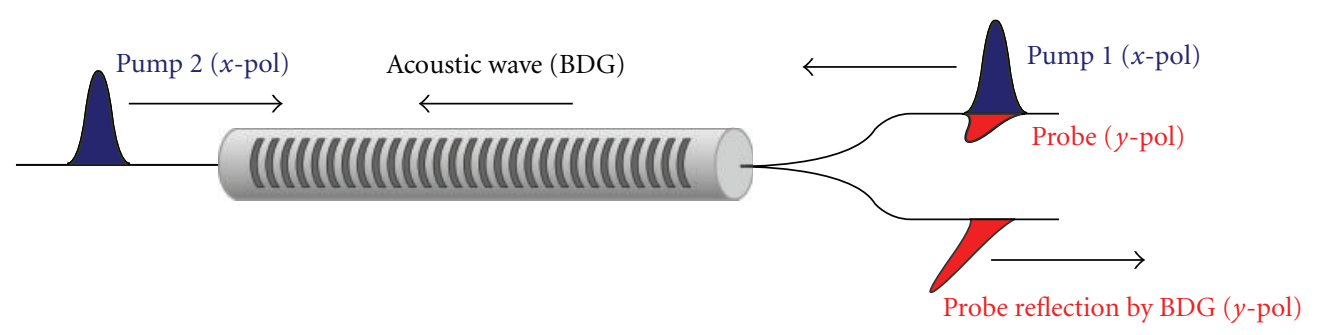

FIGURE 11: Schematic operation of BDG in an optical fiber.

distribution is extremely demanding for the performance of the BOTDR equipment, since the declared accuracy could be obtained only with a strain step length of $1 \mathrm{~m}$. However, the situation is different for smart-FRP sensor, where the bridging effect due to structural fibers "spreads" the strain peak over a certain shear stress transfer length. Both the bigger fiber length that is interested by the phenomenon and the smoother transition in the strain level contribute to enhance the strain sensitivity of smart-FRP in comparison with other fiber installation technique. From the obtained results it must be remarked that NSF took about 28 manhours to be installed on four girders, while smart-FRP took only 9 man-hours to be installed on three slabs. In addition, authors claim that NSF installation resulted to be much more problematic for the amount of dust and noise produced during groove cutting. All the results drive to the conclusion that for this kind of application in terms of performance enhancements, installation cost, and time reduction the smart-FRP system has to be considered as an optical sensor installation in bridges technique [72].

On Götaälv Bridge (built in 1939 in Sweden) severe cracking in zones above columns and a minor collapse in a structural element were observed. For safety and security reasons the bridge was monitored continuously for unusual strain changes, as well as for crack detection and localization during the refurbishing works. To do this tasks a largescale distributed fiber optic measuring system developed by Enckell et al. [73] was implemented on the structure (Figure 12).

The project was carried out with the aims of detecting and localizing cracks that may occur due to fatigue and mediocre quality of steel; to report automatically about high strain values, high strain variation as well as temperature values in short-term and long-term perspective; and to send warnings to the traffic authorities as owner of the bridge. In Figure 13 the Brillouin gain response from a fiber transducer zone with a crack is illustrated. Results of the tests are enlightened, presented, and discussed in the reference by Enkell et al. [73].

A good example of truly-distributed sensing, based on brillouin optical time domain analysis (BOTDA)-in addition to a discrete long-gauge sensing, based on Fiber Bragg-Gratings-was installed on the Streicker Bridge at Princeton University campus during its construction. The sensors were embedded in concrete during the construction. The technique allows measurements of hydration swelling and contraction in the first stage, and posttensioning of

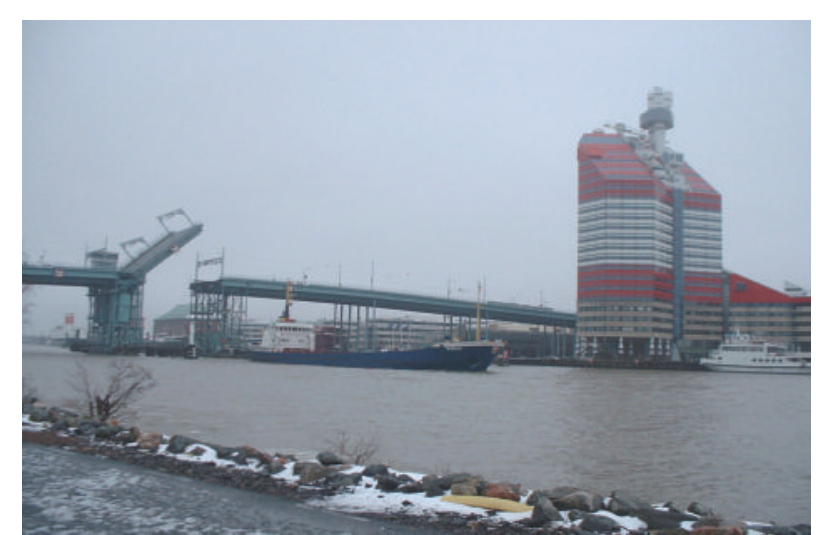

(a)

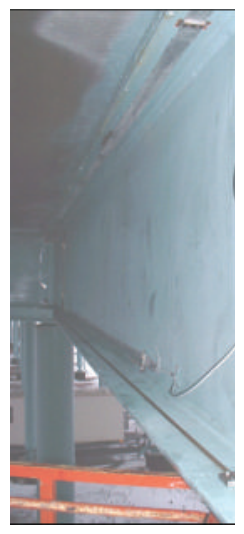

(b)

Figure 12: Götaälv Bridge with distributed Brillouin fiber sensors (a). To monitoring the structure SMARTape sensors were glued over 5 main girders ( $\sim 1000 \mathrm{~m}$ each) (b). Courtesy of Daniele Inaudi.

concrete was registered by both systems and placed side by side in order to compare their performances. Aside from the usual behavior, an unusual increase in strain was detected by several sensors in various cross-sections. The nature of this event is still under investigation, but preliminary study indicates early-age cracking as the cause. The comparison between the two monitoring systems shows good agreement in the areas where no unusual behavior was detected, but some discrepancies are noticed at locations where unusual behavior occurred and during the early age of concrete. According to the authors, these discrepancies are attributed 


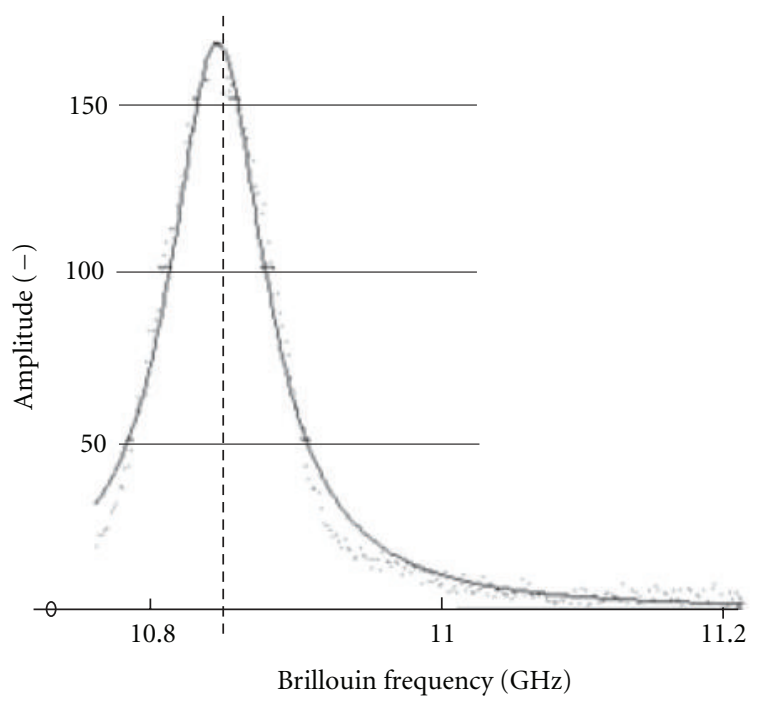

(a)

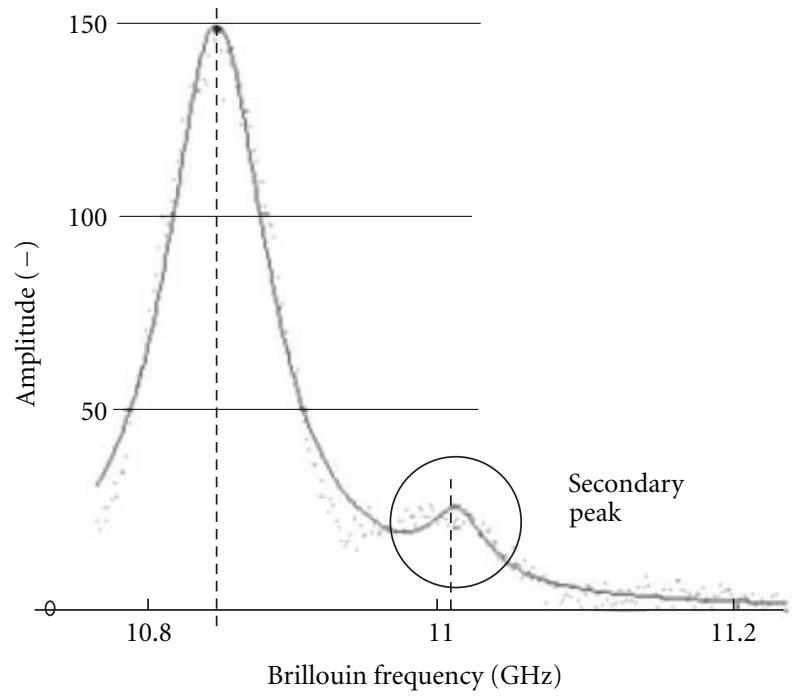

(b)

FIGURE 13: Illustration, the Brillouin gain response of fiber transducer embedded in a concrete structure with and without a crack, courtesy of Daniele Inaudy.

to the spatial resolution of the distributed monitoring system and the temperature influences at early age [74].

To measure the longitudinal strain distribution of a bridge a distributed optical fiber sensor is used [75]. A single mode optical fiber was attached on the surface using epoxy glue along the length and two ESG (electric strain gauge) were attached at a $1 / 4$ position and $1 / 2$ position of the length of the bridge, near the optical fiber. The optical fiber was connected to a developed fiber optic sensor system based on the concept of the Brillouin optical correlation domain analysis (BOCDA). The longitudinal strain of the bridge was directly measured at 195 points, and a spatial resolution of $14.5 \mathrm{~cm}$. The measurements were made for several values of loads which remains constant during a period of time (from 0 to $50 \mathrm{kN}$ ), and a proportional increasing of the load from
0 to $70 \mathrm{kN}$ at a rate of $0.33 \mathrm{kN} / \mathrm{s}$. A remarkable conclusion of this work is that the strain measured by the optical fiber and the strain distribution shape of the girder coincide with the data of ESG (electric strain gauge) within the measurement error range [75].

Recently, a study of distributed sensing on 4 span model bridge was carried out. The study consists on data analysis of gathered information from BOTDA and BOTDR systems; the project was addressed to acquire useful data to identify bridge assessment conditions [76]. It can also be mentioned that employing Brillouin fiber distributed sensing techniques, the strain distribution (with a spatial resolution of 3 meters and a resolution of $15 \mu \varepsilon$ ) along a supporting beam of a road-bridge with a span length of $44.40 \mathrm{~m}$ has been successfully carried out [77].

4.1.2. Rails Monitoring. Transverse strain of a rail can be used to determine the wheel load and lateral force, which affect the derailment coefficient and a rate of change of the wheel load. However, the longitudinal strain distribution of a rail affects on the buckling and fractures. To measure the longitudinal strain distribution of a rail section in real time, a fiber optic distributed sensor system based on the Brillouin correlation domain analysis (BOCD) was used by [78]. The experimental setup is formed by two KS60 rails with a length of $3.3 \mathrm{~m}$ fastened on five sleepers using the eCLIP fastening device, a frame to add the train load evenly to the left and right side of the rail was laid in a middle position, and two vertical load actuators were placed on the rails and coupled with a frame by bolts. A single mode optical fiber with a diameter or $250 \mu \mathrm{m}$ was attached on the surface using epoxy glue with a length of $2.8 \mathrm{~m}, 250 \mathrm{~mm}$ apart from both ends of the rail, and an electric strain gauge (ESG) was located in the span center of the rail, attached on the foot surface near the sensing optical fiber. The authors report that in the test measurement, the vertical load to each rail was increased to $143 \mathrm{kN}$ and maintained constant for $10 \mathrm{~min}$. Authors also claim that the vertical load bends the rail and that the longitudinal strain in the rail is proportional to the curvature and varies linearly with the distance from the neutral surface of the cross section. The spatial resolution for this measure was reported on $3.6 \mathrm{~cm}$ along the rail.

The Brillouin frequency distribution along the $2.8 \mathrm{~m}$ fiber (strain coefficient of $0.05 \mathrm{MHz} / \mu \varepsilon$ ) appears in a symmetrical shape with the maximum at the center position where the Brillouin frequency was reported on $10.897 \mathrm{GHz}$ with an increase about $15 \mathrm{MHz}$. Additionally, in order to validate the sensor reading, the experimental data were checked with the finite element results.

4.2. Geotechnical Structures Monitoring. To understand the bearing behavior of a loaded ground anchor, the measuring and monitoring of the stress distribution in the anchor tendon is essential. To provide this information to the geotechnical engineers, novel monitoring ground anchors using embedded optical fibers along the anchor tendon were developed using a BOTDA technique $[79,80]$. In a first step, optical sensors have been integrated into short tendons 


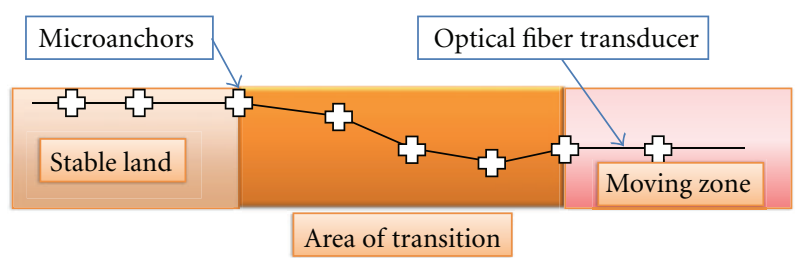

FIGURE 14: The trench cut in the hiking path: (left) attachment of the "microanchors" to the cable and, (right) compacting of the soil above the cable.

using different methods and laboratory strain testing was performed on these instrumented tendons (Figure 14). The evaluation of the laboratory testing enabled the design and development of an $8 \mathrm{~m}$ long monitoring ground anchor for field application. In 2009, this anchor has been placed into a wall supporting an excavation pit and, subsequently, anchor pullout test was carried out. The anchor was loaded stepwise up to $470 \mathrm{kN}$, almost reaching its ultimate bearing capacity. Optical measurements were taken successfully at each load step. Authors report comparison of the optical data with data acquired using conventional methods, and they claim good consistency of the results.

Geotextiles are generally used to perform functions of separation, reinforcement, filtration, and/or drainage, and they have been used to solve civil engineering problems over more than three decades. Since fabrication processes of geotextiles and optical fiber have substantially evolved, nowadays it is possible to combine both materials to produce a nonconventional optical sensor. Thus, several geotextiles with embedded optical fibers have been proposed and developed for optical sensing (Figure 15(a)). By using Brillouin interrogation techniques smart structures capable of monitoring and/or measuring the strain and/or temperature distribution in 2-dimentions can be developed. Employing geotextiles, distributed measurements of critical mechanical deformations (or soil displacements) on dikes with several kilometers length can be carried out. Additionally, they are commonly used in dikes for reinforcement of the dike body and erosion prevention, among other possibilities.

A BOFDA monitoring system has been optimized to fit the demands on dike monitoring: detection of mechanical deformation (strain) with a spatial resolution of $5 \mathrm{~m}$ over a distance range of up to $10 \mathrm{~km}$. The functionality of the monitoring system and the fiber-sensors-equipped geotextiles has been proven in several installations and field tests in dikes and dams such as the gravity dam in Solina, Poland in August 2006. A thin soil layer of several $10 \mathrm{~cm}$ put onto the geomats after installation has been proven to be a sufficient protection of the textile-integrated glass fiber cables against heavy machinery and construction work.

It must also be noticed that on the laboratory dike $(15 \mathrm{~m}$ long) at Hannover University, Germany, applications like the before tested were carried out. Sensor-based geotextiles were installed on top of the dike and were covered with a thin soil layer (Figure 15(b)). To simulate a mechanical deformation/soil displacement, a lifting bag was embedded into the soil and was inflated by air pressure. This induced

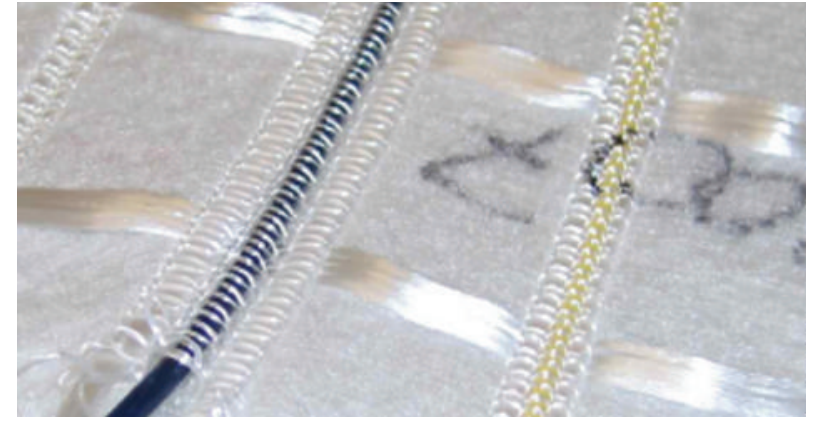

(a)

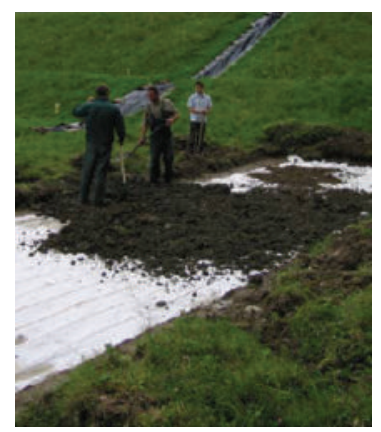

(b)

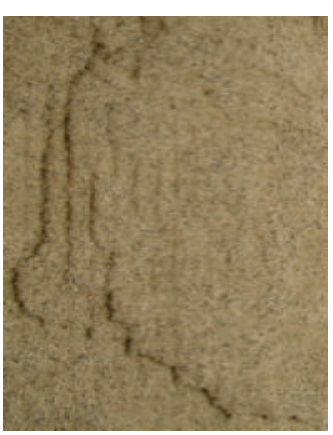

(c)
FIGURE 15: Geotextile equipped with the Fiber-ware special sensing cable and the Nexans standard indoor cable (yellow). Installation in two independent scenarios: the surface of a dike foot (b) and in a soil displacement (courtesy of K. Krebber).

a break of the inner slope of the dike and a soil displacement (Figure 15(c)). The soil displacements were clearly detected and localized by the BOFDA system [81-85].

Cross-border smuggling tunnels enable unmonitored movement of people or forbidden stuff (drugs, weapons, etc.), and they may suppose a threat to homeland security. To contribute to overcome this risk, a method for detecting the excavation of small (diameter $<1 \mathrm{~m}$ ) tunnels in clayey soils using Brillouin optical time domain reflectometry (BOTDR) and a neural network was proposed [86]. The reported architecture includes two fiber optic layouts. One is a horizontal fiber buried at a shallow depth below ground surface, and the other one has a fiber embedded in vertical minipiles to detect very deep tunnels. In both configurations, strains would develop in the fiber due to the soil displacements induced by the tunnel excavation. It was demonstrated by the authors that the proposed system was capable of detecting even small tunnel, $0.5 \mathrm{~m}$ diameter, as deep as $20 \mathrm{~m}$ under the horizontal fiber or as far as $10 \mathrm{~m}$ aside from the minipile, if the volume loss is greater than $0.5 \%$.

4.3. Pipelines Monitoring. By using Brillouin scatteringbased fiber distributed techniques, real-time monitoring and early warning systems for liquid and gas pipes have been successfully checked in real installations in a wide set of scenarios. As illustrated in Figure 16, in buried pipes and in general terms, leaks could be detected through temperature 


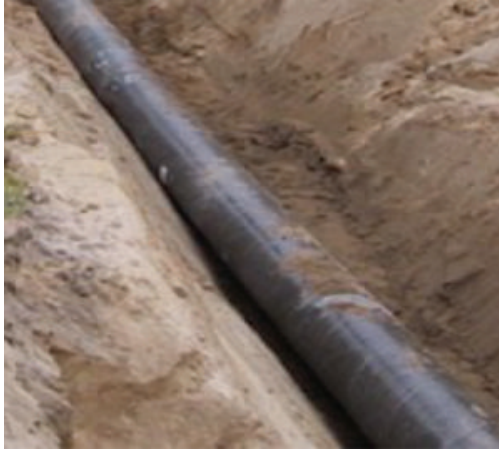

(a)

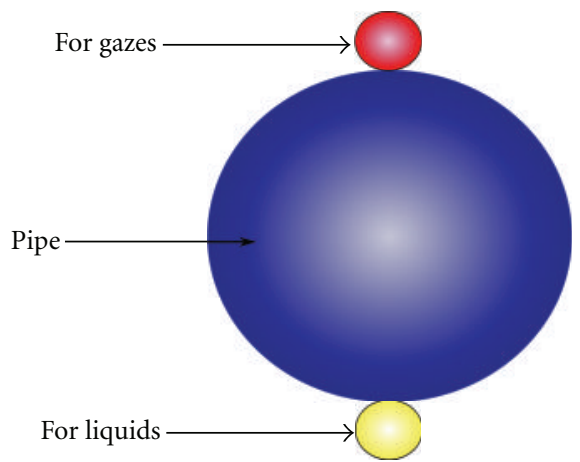

(b)

FIGURE 16: Illustration of a pipeline in a buried process (a). Scheme of the distributed fiber transducer placement with respect to the pipe section (b).

changes on the upper zone (gases) or the bellow zone (liquids) of the pipe; that is, the fiber optics transducercable of the distributed sensor system must be placed over the pipe for gases and below it for liquid leaks detection, respectively. The strain/stress state and safety of casing pipes in oil wells arenas are monitored and evaluated by using BOTDR systems such as in Daqing oilfield [87].

The brine (saturated water with salt) is a waste product derived from the mining technique for building underground caverns for gas storage in large rock-salt formation. This brine must be transported by pipelines for its processing or injected back safely into the ground. Because the brine can be harmful to the environment, pipeline small leak detection is a mandatory requirement. As in this case any change in the local temperature indicates a brine leakage in the pipeline section. As an example of this problem, a distributed temperature sensing system was integrated in the brine pipeline placed in the area of Berlin in Germany. The brine is pumped out of the underground caverns and is injected into the pipeline at a temperature of $35^{\circ} \mathrm{C}$ to $40^{\circ} \mathrm{C}$. At normal flow rate the temperature gradient along the whole pipeline length is about $8^{\circ} \mathrm{C}$. Since the pipeline is buried at a depth of approximately 2 to 3 meters, the seasonal temperature variations are quite small and the average soil temperature was measured to be around $5^{\circ} \mathrm{C}$. As a result a substantial temperature increase is associated to any pipeline leak even in the case of very small leakages [88].
Offshore arctic conditions are a challenge to the safe operation of subsea pipelines exposed to seabed ice gouging, permafrost thaw settlement, strudel scour, and channel migration. The application of fiber optic-based distributed temperature monitoring systems was carried out to monitor the pipeline operational conditions in arctic pipelines [89]. As visual inspection is impossible, real-time temperature monitoring via optical fibers along the pipeline route can provide an early warning of the development of erosional events, pipeline insulation damages, and seabed soil modifications. In order to monitor two offshore pipelines in the Alaska's Beaufort Sea oil fields a distributed temperature sensor monitoring system was implemented. The pipeline installation is part of Oooguruk oil field developments in the Beaufort Sea. It is composed of $8 \mathrm{~km}$ of buried subsea flow-lines transporting the produced fluids from an offshore gravel island/drill site to an onshore above ground pipeline which runs to an existing transmission pipeline. A total of $14 \mathrm{~km}$ of pipeline distance is continuously monitored with the fiber optic communication cables installed within the pipeline bundle. The monitoring system was demonstrated to meet the monitoring performance to detect temperature events occurring over just one meter, such as leaks and erosional events. The system has been able to map seabed temperature profiles along the pipeline route and to accurately track temperature excursions as they were occurring with field verified data prior to and during pipeline operation startup. The monitoring system operates permanently and continuously with an active leak detection system based on the detection of local temperature variations [89].

4.4. Some Materials and Structures Monitoring Cases. Applications of the Brillouin distributed sensing include a large variety of structures, such as competition yachts or experimental vehicles. Monocoque structures such as those made of carbon fiber reinforced plastic (CFRP) were instrumented with this fiber distributed technology to measure and monitor strain and temperature during manufacturing and in-service structural performance like its stiffness [90]. The sensing technique was embedded in a couple of International America's Cup Class (IACC) yachts (Asura and Idaten). The yachts were equipped with a fiber-optic distributed sensor using Brillouin scattering to monitor the longitudinal and transverse strains of the yachts to assess the stiffness of the structures in America's Cup 2000 (held at Auckland in New Zealand). Another monocoque structure is a fullscale model built as a prototype to demonstrate feasibility of a Japanese experimental reentry vehicle, namely, HOPE$\mathrm{X}$ (H-II Orbiting Plane-Experimental). The same type of sensor that was applied to the IACC yachts and a sensor using Raman scattering were used for strain and temperature measurements during a manufacturing process and for strain measurements in structural tests.

Another Brillouin-based system application with a spatial resolution of $10 \mathrm{~cm}$ was utilized for distributed strain measurement in a representative carbon fiber reinforced plastic (CFRP) stiffened panel manufactured by vacuum assisted resin transfer molding (VARTM). Strain changes 
induced during the manufacturing process and the impact tests were comprehensively presented and discussed. Carbon fiber reinforced plastic (CFRP) is being used on almost all modern commercial aircraft as a primary structural material, but it is still difficult to precisely manufacture cocured large-scale CFRP structures and ensure their structural integrity during operation. Hence there is an urgent need to develop innovative techniques to monitor the internal states of composite structures and utilize the obtained data to improve structural design, processing technologies, and maintenance methods. By combining all the information obtained by the fiber-optic network, the structural health can be accurately evaluated [91].

Honeycomb sandwich structures are integral constructions consisting of two thin facesheets and a lightweight honeycomb core. They are widely utilized in aircraft structures on account of their excellent mechanical properties. However, the lightweight structure absorbs a large amount of water while in service. Over time, cracks form in the facesheets and the adhesive layer as a result of mechanical and thermal loading, creating leak paths from the surface to the honeycomb core. Then, the water enters the core through the paths and accumulates in the cells of the honeycomb structure. Mechanical deterioration of the honeycomb core such as delamination of the face-sheet from the core can be provoked by the accumulated water. This trouble can be detected measuring the nonuniform temperature distribution induced by the water in the cell of the honeycomb core. Using this principle and a Brillouin distributed fiber optic system the water accumulation monitoring of large-scaled structures was effectively detected [92].

In large AC power generators, fatigues on electrical insulation and mechanical components could cause deterioration of the stator coil tightness and provoke a failure. Additionally, during the installation of an $\mathrm{AC}$ power generator, the fiberglass ripple spring, which is located just below the stator wedge, must be sufficiently compressed to be flat or nearly flat to ensure the maximum tightness, to compensate for the stator coil ground-wall insulation creep that is the primary factor in stator coil looseness resulting in "slot pounding". Using a DPP-BOTDA this deformation is measured [93]. The sensing fiber is glued to a fiberglass flat plate, which is subjected to a periodic side force from a fiberglass ripple spring and is consequently characterized by a periodic deformation. The measurement of the longitudinal strain of the flat plate caused by the compression of the ripple spring is performed with the Brillouin technique. Then the shape of the flat plate is reconstructed according to the strain-displacement relation. Measurements of distributed lateral displacement of the flat plate with different periods of 3 and $3.25 \mathrm{~cm}$ are reported by the authors with a maximum displacement of $0.43 \mathrm{~mm}$ and a minimum measurable displacement of $\sim 40 \mu \mathrm{m}[$ [93].

Steel corrosion has become a major problem worldwide, especially for structures exposed to aggressive environments. When corrosion happens, the volume of the steel rebar will increasingly expand due to the rust product accumulation on the surface of the steel rebar. Using this principle, a fiber BOTDA sensing technique was implemented to monitor

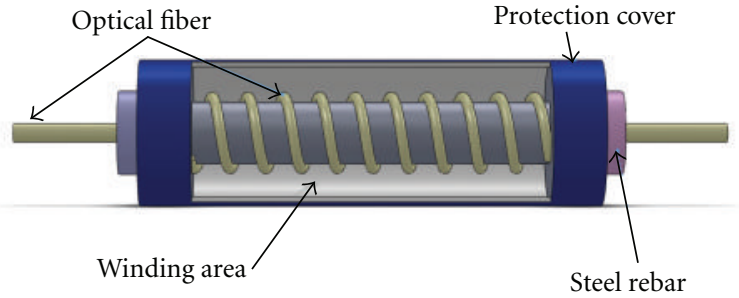

FIgURE 17: Basic packaging structure of the Brillouin corrosion expansion sensor.

corrosion expansion of steel rebars in steel reinforced concrete structures [94]. This kind of fiber optic coil winding Brillouin corrosion expansion sensors with different fiber optic coil winding packaging schemes was embedded into concrete specimens to monitor expansion strain caused by steel rebar corrosion. It was demonstrated by the authors that the expansion strain along the fiber optic coil winding area can be detected and measured using this kind of sensors during development of the corrosion. Three different types of these transducers were constructed by winding the authors around a polished steel rebar into a pretensioned fiber optic coil (Figure 17). The fiber optic will be stretched when the volume of the steel rebar expands due to corrosion and hence the tension strain change of the fiber optic coil in corrosion area can be monitored using a BOTDA analyzer.

The first kind of sensor offered a rather high sensitivity being able to detect signals of steel corrosion just at the beginning of the process. The second kind of sensor offered a relative low sensitivity compared with the first one; however, the larger monitoring range with a maximum expansion of $5690 \mu \varepsilon$ compensates for this to make it suitable for application in the resinous corrosion situations. Finally, the third kind of sensor showed the lowest sensitivity but the largest monitoring range $(6738 \mu \varepsilon)$ of the three ones. It can perform steel corrosion monitoring where there may exist an extremely corrosive environment.

\section{Summary and Conclusions}

Brillouin scattering can be successfully used to develop distributed fiber sensor systems. In these sensing approaches the optical fiber works, simultaneously, as transducer and as channel. Temperature and/or the strain Brillouin scattering dependence in conjunction with time, frequency, polarization, and others interrogation techniques, are employed to measure the change of specific parameters (measurands) along the entire fiber transducer with a given spatial resolution. Hence, the spatial resolution (minimum fiber length required to measure two consecutive perturbations or events) and the dynamic range (maximum fiber length of the transducer) are key factors for this kind of sensors.

Spontaneous and stimulated Brillouin scattering effects can be used for developing distributed fiber sensors. In the former scattering only an incident light is launched into the optical fiber and there is not any additional optical stimulus. Stimulated Brillouin sensors need additional stimulus for 
the phonon generation. Stimulated process can be obtained by an additional counter propagated light.

Brillouin distributed fiber sensors are useful for structural health monitoring systems because they can provide relevant information concerning the structural integrity of structures, even those that work in hazardous environments. Some of the most interesting applications in civil structures and in the smart materials have been briefly presented. Civil structures, bridges SHM (Götaälv Bridge, 1939 in Sweden, Streicker Bridge at Princeton University campus, among others), rails, geotechnical, pipelines structures and monitoring cases have been mentioned, as well as, distributed Brillouin sensor applications on composite materials such as carbon fiber reinforced plastic (CFRP) or honeycomb sandwichbased structures. Major problems especially for structures exposed to aggressive environments such as the measurement of fatigue on the electrical insulators, mechanical components and steel corrosion were briefly commented.

\section{Acknowledgments}

This work has been supported by the Spanish TEC201020224-C02-02 Project. Authors acknowledge Hany Shokry for his collaboration.

\section{References}

[1] J. M. Lopez-Higuera, Ed., Handbook of Optical Fibre Sensing Technology, John Wiley \& Sons, New York, NY, USA, 2002.

[2] D. Leandro, A. Ullan, A. Loayssa, J. M. López-Higuera, and M. López-Amo, "Remote $(155 \mathrm{~km})$ fiber bragg grating interrogation technique combining Raman, Brillouin, and erbium gain in a fiber laser," IEEE Photonics Technology Letters, vol. 23, no. 10, pp. 621-623, 2011.

[3] C. Galindez, F. Madruga, A. Ullan, and J. M. Lopez-Higuera, "Técnica y dispositivo para conformar el espectro de ganancia de Brillouin en guías de onda ópticas,” Patent, 2009.

[4] A. Rogers, "Distributed optical-fibre sensing," in Handbook of Optical Fibre Sensing Technology, J. M. Lopez-Higuera, Ed., chapter 14, John Wiley \& Sons, New York, NY, USA, 2002.

[5] G. P. Agrawal, Nonlinear Fiber Optics, Quantum ElectronicsPrinciples and Applications, Academic Press, Rochester, NY, USA, 1995.

[6] M. Niklès, L. Thévenaz, and P. A. Robert, "Brillouin gain spectrum characterization in single-mode optical fibers," Journal of Lightwave Technology, vol. 15, no. 10, pp. 1842-1851, 1997.

[7] R. W. Boyd, Nonlinear Optics, Academic Press, Rochester, NY, USA, 2nd edition, 2003.

[8] T. Horiguchi, K. Shimizu, T. Kurashima, M. Tateda, and Y. Koyamada, "Development of a distributed sensing technique using Brillouin scattering," Journal of Lightwave Technology, vol. 13, no. 7, pp. 1296-1302, 1995.

[9] T. Kurashima, T. Horiguchi, and M. Tateda, "Distributedtemperature sensing using stimulated Brillouin scattering in optical silica fibers," Optics Letters, vol. 15, no. 18, pp. 10381040, 1990.

[10] C. A. Galindez, F. J. Madruga, M. Lomer, A. Cobo, and J. M. Lopez-Higuera, "Effect of humidity on optical fiber distributed sensor based on Brillouin scattering," in Proceedings of the 19th International Conference on Optical Fibre Sensors (OFS '08), vol. 70044W, pp. 70041-70044, April 2008.
[11] C. Galindez, F. J. Madruga, and J. M. Lopez-Higuera, "Influence of humidity on the measurement of Brillouin frequency shift," IEEE Photonics Technology Letters, vol. 20, no. 23, pp. 1959-1961, 2008.

[12] C. Galindez, F. J. Madruga, and J. M. Lopez-Higuera, "Brillouin frequency shift of standard optical fibers set in water vapor medium," Optics Letters, vol. 35, no. 1, pp. 28-30, 2010.

[13] D. Culverhouse, F. Farahi, C. N. Pannell, and D. A. Jackson, "Potential of stimulated Brillouin scattering as sensing mechanism for distributed temperature sensors," Electronics Letters, vol. 25, no. 14, pp. 913-915, 1989.

[14] M. N. Alahbabi, Y. T. Cho, and T. P. Newson, "150 km-range distributed temperature sensor based on coherent detection of spontaneous Brillouin backscatter and in-line Raman amplification," Journal of the Optical Society of America B, vol. 22, no. 6, pp. 1321-1324, 2005.

[15] M. Alahbabi, Y. T. Cho, and T. P. Newson, "Comparison of the methods for discriminating temperature and strain in spontaneous Brillouin-based distributed sensors," Optics Letters, vol. 29, no. 1, pp. 26-28, 2004.

[16] Y. T. Cho, M. Alahbabi, M. J. Gunning, and T. P. Newson, " $50 \mathrm{~km}$ single-ended spontaneous-Brillouin-based distributed-temperature sensor exploiting pulsed Raman amplification," Optics Letters, vol. 28, no. 18, pp. 1651-1653, 2003.

[17] S. M. Maughan, H. H. Kee, and T. P. Newson, " $57 \mathrm{~km}$ single-ended spontaneous Brillouin-based distributed fiber temperature sensor using microwave coherent detection," Optics Letters, vol. 26, no. 6, pp. 331-333, 2001.

[18] M. N. Alahbabi, Y. T. Cho, and T. P. Newson, " $100 \mathrm{~km}$ distributed temperature sensor based on coherent detection of spontaneous Brillouin backscatter," Measurement Science and Technology, vol. 15, no. 8, pp. 1544-1547, 2004.

[19] T. Kurashima, T. Horiguchi, H. Hizumita, S. Furukawa, and Y. Koyamada, "Brillouin optical-fiber time domain reflectometry," in Proceedings of the International Quantum Electronics Conference, Dig Tech, Vienna, Austria, 1992.

[20] Y. Lu, H. Liang, X. Zhang, and F. Wang, "Brillouin optical time-domain reflectometry based on Hadamard sequence probe pulse," in Proceedings of the 9th International Conference on Optical Communications and Networks (ICOCN '10), pp. 36-38, October 2010.

[21] P. C. Wait and T. P. Newson, "Landau Placzek ratio applied to distributed fibre sensing," Optics Communications, vol. 122, no. 4-6, pp. 141-146, 1996.

[22] Y. Mizuno, W. Zou, Z. He, and K. Hotate, "Proposal and experiment of BOCDR-Brillouin optical correlation-domain reflectometry," in Proceedings of the International Society for Optical Engineering (SPIE '08), University of Western Australia, Optical and Biomedical Engineering Laboratory (OBEL), April 2008.

[23] Y. Mizuno, Z. He, and K. Hotate, "Measurement range enlargement in Brillouin optical correlation-domain reflectometry based on temporal gating scheme," Optics Express, vol. 17, no. 11, pp. 9040-9046, 2009.

[24] T. Horiguchi and M. Tateda, "BOTDA-nondestructive measurement of single-mode optical fiber attenuation characteristics using Brillouin interaction: theory," Journal of Lightwave Technology, vol. 7, no. 8, pp. 1170-1176, 1989.

[25] D. Iida and F. Ito, "Detection sensitivity of Brillouin scattering near Fresnel reflection in BOTDR measurement," Journal of Lightwave Technology, vol. 26, no. 4, pp. 417-424, 2008.

[26] C. A. Galindez and L. Thevenaz, "Effect of pulse chirp on distributed Brillouin fiber sensing," in Proceedings of the 19th SPIE International Conference on Optical Fibre Sensors 
(OFS '08), vol. 7004, pp. 70041J-70044J, SPIE-The International Society for Optical Engineering, Perth, Australia, 2008.

[27] V. Lecoeuche, D. J. Webb, C. N. Pannell, and D. A. Jackson, "Transient response in high-resolution Brillouin-based distributed sensing using probe pulses shorter than the acoustic relaxation time," Optics Letters, vol. 25, no. 3, pp. 156-158, 2000.

[28] C. Galindez, F. J. Madruga, A. Cobo, O. Conde, and J. M. Lopez-Higuera, "Pulse shape effects on the measurement of Temperature using a Brillouin based Optical fiber sensor," in Proceedings of the Enabling Photonics Technologies for Defense, Security, and Aerospace Applications III (EWOFS '07), vol. 65720, pp. 65721-65724, April 2007.

[29] H. Naruse and M. Tateda, "Optimum temporal pulse shape of launched light for optical time domain reflectometry type sensors using Brillouin backscattering," Optical Review, vol. 8, no. 2, pp. 126-132, 2001.

[30] K. Kishida and C. -H. Li, "Pulse pre-pump-BOTDA technology for new generation of distributed strain measuring system," in Structural Health Monitoring and Intelligent Infrastructure, pp. 471-477, Taylor \& Francis, London, UK, 2006.

[31] C. A. Galindez, A. Quintela, M. A. Quintela, and J. M. LopezHiguera, " $30 \mathrm{~cm}$ of spatial resolution using pre-excitation pulse BOTDA technique," in Proceedings of the 21st International Conference on Optical Fiber Sensors (OFS '11), vol. 77532, pp. 77531-77534, May 2011.

[32] C. A. Galindez, A. Quintela, M. A. Quintela, and J. M. LopezHiguera, " $30 \mathrm{~cm}$ of spatial resolution using pre-excitation pulse BOTDA technique," in Proceedings of the 21st International Conference on Optical Fiber Sensors, pp. 2344-2348, May 2011.

[33] L. Thevenaz and S. F. Mafang, "Distributed fiber sensing using Brillouin echoes," in Proceedings of the 19th International Conference on Optical Fibre Sensors (SPIE '08), pp. 70043N770044.

[34] W. Li, X. Bao, Y. Li, and L. Chen, "Differential pulse-width pair BOTDA for high spatial resolution sensing," Optics Express, vol. 16, no. 26, pp. 21616-21625, 2008.

[35] S. M. Foaleng, M. Tur, J. C. Beugnot, and L. Thévenaz, "High spatial and spectral resolution long-range sensing using Brillouin echoes," Journal of Lightwave Technology, vol. 28, no. 20, Article ID 5565369, pp. 2993-3003, 2010.

[36] A. W. Brown, B. G. Colpitts, and K. Brown, "Distributed sensor based on dark-pulse Brillouin scattering," IEEE Photonics Technology Letters, vol. 17, no. 7, pp. 1501-1503, 2005.

[37] Y. Dong, H. Zhang, L. Chen, and X. Bao, " $2 \mathrm{~cm}$ spatialresolution and $2 \mathrm{~km}$ range Brillouin optical fiber sensor using a transient differential pulse pair," Applied Optics, vol. 51, no. 9, pp. 1229-1235, 2012.

[38] T. Sperber, A. Eyal, M. Tur, and L. Thévenaz, "High spatial resolution distributed sensing in optical fibers by Brillouin gain-profile tracing," Optics Express, vol. 18, no. 8, pp. 8671$8679,2010$.

[39] K. Hotate, "Measurement of brillouin gain spectrum distribution along an optical fiber using a correlation-based technique-proposal, experiment and simulation-," IEICE Transactions on Electronics, vol. E83-C, no. 3, pp. 405-411, 2000.

[40] A. Minardo, R. Bernini, and L. Zeni, "Differential techniques for high-resolution BOTDA: an analytical approach," IEEE Photonics Technology Letters, vol. 24, no. 15, pp. 1295-1297, 2012.
[41] L. Thévenaz, S. M. Foaleng, K. Y. Song et al., "Advanced Brillouin-based distributed optical fibre sensors with submeter scale spatial resolution," in Proceedings of the 36th European Conference and Exhibition on Optical Communication (ECOC'10), pp. 1-6, September 2010.

[42] J. C. Beugnot, M. Tur, S. F. Mafang, and L. Thévenaz, "Distributed Brillouin sensing with sub-meter spatial resolution: modeling and processing," Optics Express, vol. 19, no. 8, pp. 7381-7397, 2011.

[43] A. Minardo, R. Bernini, and L. Zeni, "Spatial resolution enhancement in preactivated BOTDA schemes by numerical processing," IEEE Photonics Technology Letters, vol. 24, no. 12, pp. 1003-1005, 2012.

[44] M. N. Alahbabi, Y. T. Cho, T. P. Newson, P. C. Wait, and A. H. Hartog, "Influence of modulation instability on distributed optical fiber sensors based on spontaneous Brillouin scattering," Journal of the Optical Society of America B, vol. 21, no. 6, pp. 1156-1160, 2004.

[45] Y. Dong, L. Chen, and X. Bao, "System optimization of a longrange Brillouin-loss-based distributed fiber sensor," Applied Optics, vol. 49, no. 27, pp. 5020-5025, 2010.

[46] M. A. Soto, G. Bolognini, and F. Di Pasquale, "Analysis of optical pulse coding in spontaneous Brillouin-based distributed temperature sensors," Optics Express, vol. 16, no. 23, pp. 19097-19111, 2008.

[47] R. Bernini, A. Minardo, and L. Zeni, "Long-range distributed Brillouin fiber sensors by use of an unbalanced double sideband probe," Optics Express, vol. 19, no. 24, pp. 23845-23856, 2011.

[48] M. Soto, M. Taki, G. Bolognini, and F. Di Pasquale, "Optimization of a DPP-BOTDA sensor with $25 \mathrm{~cm}$ spatial resolution over $60 \mathrm{~km}$ standard single-mode fiber using Simplex codes and optical pre-amplification," Optics Express, vol. 20, no. 7, pp. 6860-6869, 2012.

[49] M. A. Soto, G. Bolognini, and F. Di Pasquale, "Optimization of long-range BOTDA sensors with high resolution using firstorder bi-directional Raman amplification," Optics Express, vol. 19, no. 5, pp. 4444-4457, 2011.

[50] A. Zornoza, R. A. Pérez-Herrera, C. Elosúa et al., "Long-range hybrid network with point and distributed Brillouin sensors using Raman amplification," Optics Express, vol. 18, no. 9, pp. 9531-9541, 2010.

[51] X. Bao and L. Chen, "Recent progress in distributed fiber optic sensors,” Sensors, vol. 12, no. 7, pp. 8601-8639, 2012.

[52] Z. Liu, G. Ferrier, X. Bao, X. Zeng, Q. Yu, and A. Kim, "Brillouin scattering based distributed fiber optic temperature sensing for fire detection," in Proceedings of The 7th International Symposium on Fire Safety Conference, Worcester, Mass, USA, 2002.

[53] Y. Peled, A. Motil, L. Yaron, and M. Tur, "Slope-assisted fast distributed sensing in optical fibers with arbitrary Brillouin profile," Optics Express, vol. 19, no. 21, pp. 19845-519854, 2011.

[54] R. Bernini, A. Minardo, and L. Zeni, "Dynamic strain measurement in optical fibers by stimulated brillouin scattering," Optics Letters, vol. 34, no. 17, pp. 2613-2615, 2009.

[55] X. Bao, C. Zhang, W. Li, M. Eisa, S. El-Gamal, and B. Benmokrane, "Monitoring the distributed impact wave on a concrete slab due to the traffic based on polarization dependence on stimulated Brillouin scattering," Smart Materials and Structures, vol. 17, no. 1, Article ID 015003, 2008.

[56] P. Chaube, B. G. Colpitts, D. Jagannathan, and A. W. Brown, "Distributed fiber-optic sensor for dynamic strain 
measurement," IEEE Sensors Journal, vol. 8, no. 7, pp. 10671072, 2008.

[57] C. A. Galindez, F. J. Madruga, and J. M. Lopez-Higuera, "Efficient dynamic events discrimination technique for fiber distributed Brillouin sensors," Optics Express, vol. 19, no. 20, pp. 7-5, 1891.

[58] Y. Peled, A. Motil, and M. Tur, "Fast Brillouin optical time domain analysis for dynamic sensing," Optics Express, vol. 20, no. 8, pp. 8584-8591, 2012.

[59] K. Y. Song, Z. He, and K. Hotate, "Distributed strain measurement with millimeter-order spatial resolution based on Brillouin optical correlation domain analysis," Optics Letters, vol. 31, no. 17, pp. 2526-2528, 2006.

[60] M. Belal and T. P. Newson, "Evaluation of a high spatial resolution temperature compensated distributed strain sensor using a temperature controlled strain rig," in Proceedings of the 21st International Conference on Optical Fiber Sensors, vol. 36, pp. 4728-4730, May 2011.

[61] D. Garus, K. Krebber, F. Schliep, and T. Gogolla, “Distributed sensing technique based on Brillouin optical-fiber frequencydomain analysis," Optics Letters, vol. 21, no. 17, pp. 1402-1404, 1996.

[62] D. Garus, T. Gogolla, K. Krebber, and F. Schliep, "Brillouin optical-fiber frequency-domain analysis for distributed temperature and strain measurements," Journal of Lightwave Technology, vol. 15, no. 4, pp. 654-662, 1997.

[63] R. Bernini, A. Minardo, and L. Zeni, "Accurate high-resolution fiber-optic distributed strain measurements for structural health monitoring," Sensors and Actuators A, vol. 134, no. 2, pp. 389-395, 2007.

[64] K. Y. Song, W. Zou, Z. He, and K. Hotate, "All-optical dynamic grating generation based on Brillouin scattering in polarization-maintaining fiber," Optics Letters, vol. 33, no. 9, pp. 926928, 2008.

[65] K. Y. Song, "Operation of Brillouin dynamic grating in singlemode optical fibers," Optics Letters, vol. 36, no. 23, pp. 46864688, 2011.

[66] W. Zou, Z. He, K. Y. Song, and K. Hotate, "Correlation-based distributed measurement of a dynamic grating spectrum generated in stimulated Brillouin scattering in a polarizationmaintaining optical fiber," Optics Letters, vol. 34, no. 7, pp. 1126-1128, 2009.

[67] W. W. Zou, Z. He, and K. Hotate, "Complete discrimination of strain and temperature using Brillouin frequency shift and birefringence in a polarization-maintaining fiber," Optics Express, vol. 17, no. 3, pp. 1248-1255, 2009.

[68] Y. Dong, L. Chen, and X. Bao, "Truly distributed birefringence measurement of polarization-maintaining fibers based on transient Brillouin grating," Optics Letters, vol. 35, no. 2, pp. 193-195, 2010.

[69] K. Y. Song, K. Lee, and S. B. Lee, “Tunable optical delays based on Brillouin dynamic grating in optical fibers," Optics Express, vol. 17, no. 12, pp. 10344-10349, 2009.

[70] K. Y. Song, W. Zou, Z. He, and K. Hotate, "Optical timedomain measurement of Brillouin dynamic grating spectrum in a polarization-maintaining fiber," Optics Letters, vol. 34, no. 9, pp. 1381-1383, 2009.

[71] J. M. Lopez-Higuera, L. Rodriguez Cobo, A. Quintela Incera, and A. Cobo, "Fiber optic sensors in structural health monitoring," Journal of Lightwave Technology, vol. 29, no. 4, pp. 587-608, 2011.

[72] F. Bastianini, A. Rizzo, N. Galati, U. Deza, and A. Nanni, "Discontinuous Brillouin strain monitoring of small concrete bridges: comparison between near-to-surface and "smart"
FRP fiber installation techniques," in Proceedings of the Smart Structures and Materials 2005: Sensors and Smart Structures Technologies for Civil, Mechanical, and Aerospace Systems (SPIE '05), pp. 612-623, March 2005.

[73] M. Enckell, B. Glisic, F. Myrvoll, and B. Bergstrand, "Evaluation of a large-scale bridge strain, temperature and crack monitoring with distributed fibre optic sensors," Journal of Civil Structural Health Monitoring, vol. 1, no. 1, pp. 37-46, 2011.

[74] B. Glisic, J. Chen, and D. Hubbell, "Streicker Bridge: a comparison between Bragg-grating long-gauge strain and temperature sensors and Brillouin scattering-based distributed strain and temperature sensors," in Proceedings of the Sensors and Smart Structures Technologies for Civil, Mechanical, and Aerospace Systems, pp. 79810-79812, March 2011.

[75] H.-J. Yoon, K.-Y. Song, H.-M. Kim, and J.-S. Kim, "Strain monitoring of composite steel girder bridge using distributed optical fibre sensor system," in Procedia Engineering, pp. 25442547, Elsevier, New York, NY, USA, 2011.

[76] I.-B. Kwon, M. Malekzadeh, Q. Ma et al., "Fiber optic sensor installation for monitoring of 4 span model bridge in UCF," in Proceedings of the 29th IMAC, a Conference on Structural Dynamics, pp. 383-388, February 2011.

[77] A. Minardo, R. Bernini, and L. Zeni, "Vectorial dislocation monitoring of pipelines by use of Brillouin-based fiber-optics sensors," Smart Materials and Structures, vol. 17, no. 1, Article ID 015006, 2008.

[78] H.-J. Yoon, K. Y. Song, J. S. Kim, and D. S. Kim, "Longitudinal strain monitoring of rail using a distributed fiber sensor based on Brillouin optical correlation domain analysis," NDT and E International, vol. 44, no. 7, pp. 637-644, 2011.

[79] M. Iten and A. M. Puzrin, "Monitoring of stress distribution along a ground anchor using BOTDA," in Proceedings of the Sensors and Smart Structures Technologies for Civil, Mechanical, and Aerospace Systems, vol. 76415, p. 76472, March 2010.

[80] M. Iten, D. Hauswirth, and A. M. Puzrin, "Distributed fiber optic sensor development, testing and evaluation for geotechnical monitoring applications," in Proceedings of the Smart Sensor Phenomena, Technology, Networks, and Systems, pp. 798207-798215, March 2011.

[81] N. Noether, A. Wosniok, K. Krebber, and E. Thiele, "Dike monitoring using fiber sensor-based geosynthetics," in Proceedings of the III Eccomas Thematic Conference on Smart Structures and Materials, 2007.

[82] N. Nöther, A. Wosniok, K. Krebber, and E. Thiele, "A distributed fiber optic sensor system for dike monitoring using Brillouin optical frequency domain analysis," in Proceedings of the Smart Sensor Phenomena, Technology, Networks, and Systems, vol. 6933, pp. 69330T-69331-69330T-69339, March 2008.

[83] N. Nöther, A. Wosniok, K. Krebber, and E. Thiele, "A distributed fiber optic sensor system for dike monitoring using Brillouin optical frequency domain analysis," in Proceedings of the Smart Sensor Phenomena, Technology, Networks, and Systems, p. 700303, March 2008.

[84] N. Noether, A. Wosniok, K. Krebber, and E. Thiele, "A distributed fiber-optic sensor system for monitoring of large geotechnical strutures," in Proceedings of the 4th International Conference on Structural Health Monitoring on Intelligent Infrastructure (SHMII '09), 2009.

[85] A. Wosniok, N. Noether, K. Krebber, and E. Thiele, "Distributed monitoring of mechanical deformation in river dikes," in Proceedings of the Eurosensors XXIII conference, 2008. 
[86] A. Klar and R. Linker, "Feasibility study of automated detection of tunnel excavation by Brillouin optical time domain reflectometry," Tunnelling and Underground Space Technology, vol. 25, no. 5, pp. 575-586, 2010.

[87] Z. Zhou, J. He, M. Huang, J. He, J. Ou, and G. Chen, "Casing pipe damage detection with optical fiber sensors: a case study in oil well constructions," in Proceedings of the International Society for Optical Engineering (SPIE '10), pp. 764908-764911, March 2010.

[88] M. Nikles, "Long-distance fiber optic sensing solutions for pipeline leakage, intrusion and ground movement detection," in Proceedings of the 6th Fiber Optic Sensors and Applications (SPIE '09), pp. 731602-731613, April 2009.

[89] B. Eisler, G. Lanan, M. Niklès, and L. Zuckerman, “Distributed fiber optic temperature sensing system for buried subsea arctic pipelines," in Proceedings of the Deep Offshore Technology Conference 2008 (DOT '08), Houston, Tex, USA, 2008.

[90] H. Murayama, K. Kageyama, H. Naruse, A. Shimada, and K. Uzawa, "Application of fiber-optic distributed sensors to health monitoring for full-scale composite structures," Journal of Intelligent Material Systems and Structures, vol. 14, no. 1, pp. 3-13, 2003.

[91] S. Minakuchi, N. Takeda, S. I. Takeda, Y. Nagao, A. Franceschetti, and X. Liu, "Life cycle monitoring of largescale CFRP VARTM structure by fiber-optic-based distributed sensing," Composites A, vol. 42, no. 6, pp. 669-676, 2011.

[92] S. Minakuchi, H. Tsukamoto, and N. Takeda, "Detecting water accumulation in honeycomb sandwich structures by opticalfiber-based distributed temperature measurement," Journal of Intelligent Material Systems and Structures, vol. 20, no. 18, pp. 2249-2255, 2009.

[93] D. Yongkang, B. Xiaoyi, and C. Liang, "Online monitoring of the distributed lateral displacement in large AC power generators using a high spatial resolution Brillouin optical fiber sensor," Smart Materials and Structures, vol. 20, no. 11, pp. 115001-115006, 2011.

[94] X. Zhao, P. Gong, G. Qiao, J. Lu, X. Lv, and J. Ou, "Brillouin corrosion expansion sensors for steel reinforced concrete structures using a fiber optic coil winding method," Sensors, vol. 11, no. 11, pp. 10798-10819, 2011. 

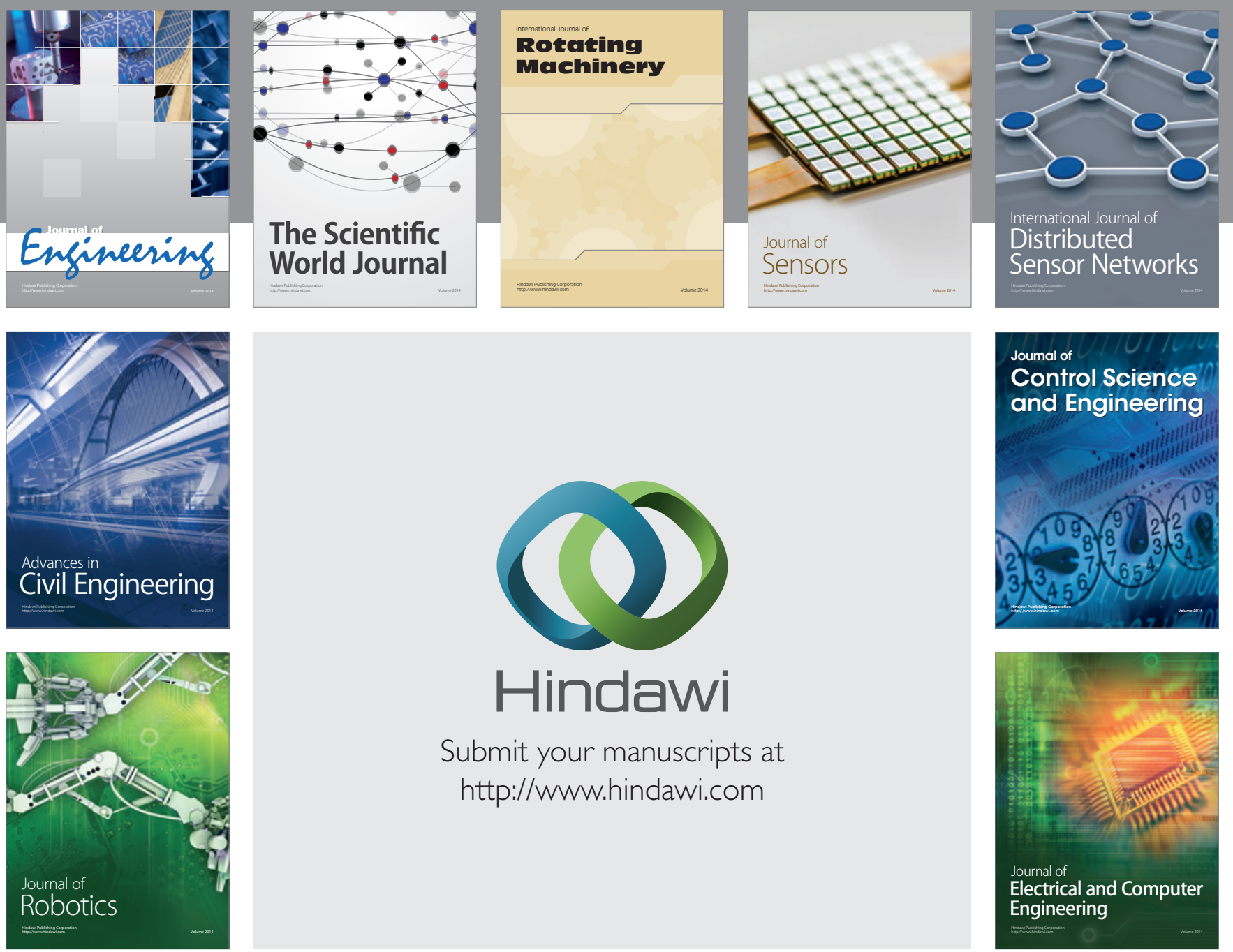

Submit your manuscripts at

http://www.hindawi.com
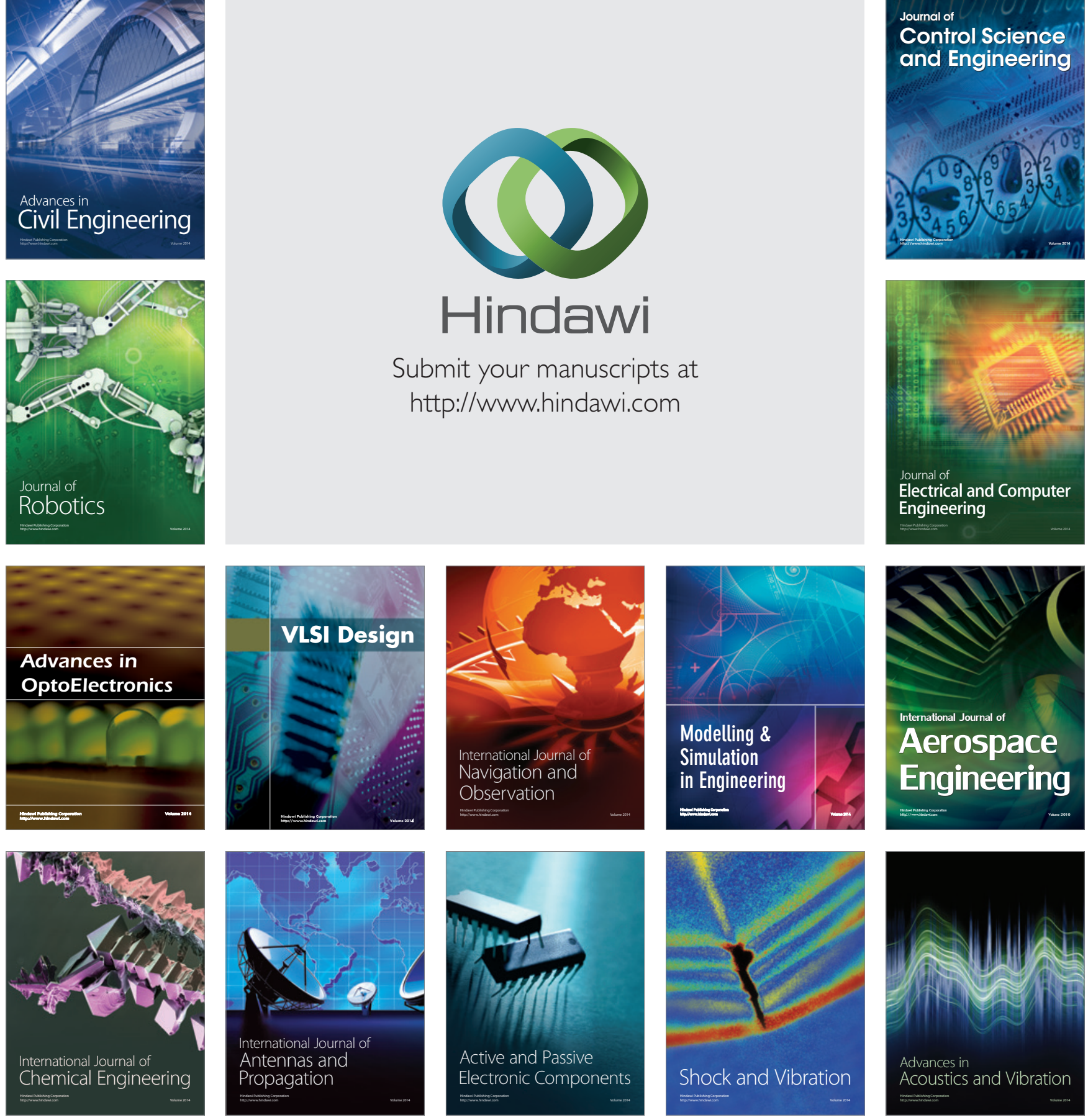